\title{
Supersonic Combustion of Pylon-Injected Hydrogen in High-Enthalpy Flow with Imposed Vortex Dynamics
}

\author{
Fabrizio Vergine, $\stackrel{*}{-}$ Matthew Crisanti,,$*$ and Luca Maddalena \\ University of Texas at Arlington, Arlington, Texas 76019 \\ Victor Miller $\stackrel{ \pm}{\underline{*}}$ \\ Stanford University, Stanford, California 94305 \\ and \\ Mirko Gamba \\ University of Michigan, Ann Arbor, Michigan 48109 \\ DOI: $10.2514 / 1 . B 35330$
}

\begin{abstract}
The ignition and combustion characteristics of the hydrogen plume issued from two pylon-type injectors in a Mach 2.4, high-enthalpy airflow are presented. Specifically, the focus of the study is on the effects of the imposed interaction and subsequent dynamics of a system of selected supersonic streamwise vortices on the reacting plume morphology and its evolution. The design phase of the experimental campaign was carried out with a reduced-order model, with the goal of identifying peculiar interactions among streamwise vortical structures introduced in the flow of interest. Two vortex interaction modes have been selected and later implemented using ramp-type vortex generators positioned symmetrically and asymmetrically on the pylon injectors, as prescribed by the results of the simulations reported here. Hydrogen/air combustion experiments, aimed at investigating the selected cases, were conducted in the expansion tube facility of the High-Temperature Gas Dynamics Laboratory at Stanford University. Stagnation enthalpy of $2.8 \mathrm{MJ} / \mathrm{kg}$, static temperature of $1400 \mathrm{~K}$, and static pressure of $40 \mathrm{kPa}$ were the chosen test conditions. Hydrogen at $300 \mathrm{~K}$ was delivered with two different total injection pressures. The supersonic combustion, ignition, and flameholding characteristics were documented with schlieren photography, $\mathrm{OH}^{*}$ chemiluminescence, and instantaneous $\mathrm{OH}$ radicals planar laser-induced fluorescence. The evolution of the reactive vortical system was probed in cross-sectional planes at a distance of $1.8,4.3,7.6$, and $10.7 \mathrm{~cm}$ from the fuel exit plane. The results are thoroughly analyzed and the marked difference in the plume morphology between the two cases is explained. The similarities between the injectant's predicted plumes and the measured distribution of $\mathrm{OH}$ radicals across the surveyed planes allow for an interpretation of the results based on supersonic vortex dynamics considerations.
\end{abstract}

\section{Introduction}

$\mathbf{T}$ HE effectiveness of supersonic combustion is a fundamental, enabling aspect for the development of airbreathing hypersonic vehicles. Decades of studies have been devoted to this matter in an attempt to gain fundamental understanding and, consequently, control authority on the combustion processes in scramjet engines. However, many complications still arise from the unpredictability of the involved phenomena when designing a practical scramjet combustor. Among the challenges to face is the ability to achieve a sufficient plume spreading for a more uniform delivery of fuel across the cross section of the combustion chamber.

In their work, Papamoschou and Roshko [1] showed the reduced capability of supersonic, planar free shear layers to grow compared with their subsonic counterparts due to compressibility effects associated with the high convective Mach number of the large-scale structures present in these flows. This suggests that a mixing strategy only based on planar layers cannot be effective for aiming at short engine lengths at high convective Mach numbers.

Studies originally conducted by Chigier and Chervinsky [2] showed that the introduction of swirl in a jet drastically increases the

Received 29 January 2014; revision received 2 April 2014; accepted for publication 3 May 2014; published online 12 August 2014. Copyright $\odot 2014$ by the American Institute of Aeronautics and Astronautics, Inc. All rights reserved. Copies of this paper may be made for personal or internal use, on condition that the copier pay the $\$ 10.00$ per-copy fee to the Copyright Clearance Center, Inc. 222 Rosewood Drive, Danvers, MA 01923; include the code 1533-3876/14 and $\$ 10.00$ in correspondence with the CCC.

* Graduate Research Assistant. Student Member AIAA.

${ }^{\dagger}$ Assistant Professor, Mechanical and Aerospace Engineering Department. Senior Member AIAA.

${ }^{\ddagger}$ Graduate Research Assistant, Mechanical Engineering Department. Student Member AIAA.

${ }^{\S}$ Assistant Professor, Aerospace Engineering Department, 530 South State Street. Member AIAA. entrainment of surrounding fluid in the plume compared with nonswirling jets and, later, Swithenbank and Chigier [3] discussed the effectiveness of swirling jets in increasing the production of turbulence and in potentially enhancing mixing with the surrounding fluid in compressible flows. More recent numerical and experimental works showed that coherent streamwise structures introduced in supersonic mixing flows increase the mixing rates between the reactants $[4,5]$ and are able to augment the growth rate of mixing layers due to additional entrainment of fluid [6] and low sensitivity to compressibility effects. Mixing enhancement with streamwise vortices is, in fact, achieved by means of the synergistic action of turbulence, strain rates at the interface between the reactants [7], and the entrainment of the right proportions of air and injectant.

Among the simplest ways to generate vorticity in both subsonic and supersonic flows is the use of contoured devices like ramps, which create a streamwise counter-rotating vortex pair (CVP) due to the so-called cross-stream shear [8]. Other possible streamwise vortices production mechanisms include the vorticity generation in a jet issued into a cross stream [9-11] and the coalescence of baroclinically generated vorticity [12]. However, regardless of the mechanism of vorticity generation, one peculiar characteristic of both incompressible and compressible vortices is their mutual interaction capability, which is responsible for the variation of their spatial displacement in the flowfield [13].

In the presence of a nearby injection plume, streamwise vortices can drastically impact the mixing between the freestream and the injectant due to a number of possible interaction modes. The very first action of these vortices is to change the morphology of the plume due to the imposed flowfield, as has been already proved in supersonic cold flow studies conducted by the authors $[14,15]$. In those works it was pointed out that, for the flow to be dominated and, therefore, controlled by the streamwise vortices, their strength must be larger than that of the spanwise structures convecting in the close layer in which they evolve. However, although notable flow physics 
downstream of different injection configurations were discovered in supersonic cold flow, studies of injection devices suitable to ultimately enhance mixing in high-enthalpy airflow are necessary to verify that similar phenomena can be obtained in both regimes.

Pylon injectors [16-18] have lately been considered as possible candidates as fueling devices suitable for large-scale scramjet engines due to their ability to introduce the fuel into the core of the incoming flow. To enhance the fuel dispersion and mixing, the integration of streamwise vortex generators on pylon and strut injector configurations has been, in recent years, the subject of extensive research, which has provided some partial indication on the possibility to relate some flow evolution phenomena to the presence of an organized rotational motion in the plume. Specifically, Doster et al. [18] published a study of three pylon injectors equipped with different ramp geometries, showing NO planar laser-induced fluorescence (PLIF) and in-stream probing measurements of the fuel plumes issuing in a Mach 2 cold flow. The investigation was principally centered on the comparison of the performance of the pylons in terms of total pressure losses and mixing levels achieved in the test, but no attempt was made to understand how the interaction among the vortical structures modified the issued plume. A joint experimental campaign between the Japan Aerospace Exploration Agency and ONERA, presented by Sunami et al. [19], involved the study of the combustion of hydrogen injected from different strut injectors, some of which were equipped with vortex-generating ramps, whereas others were ramp free. In those results, it was shown that the plume issuing from the struts furnished with swept and cantilevered ramps was clearly shaped by streamwise coherent structures, at least in its early development. However, despite the major modifications that are introduced in the plume by the vortices, in both works, the design of the injectors was not optimized to target the study on any peculiar flow feature.

For this reason, this effort is intended to explore, in a reacting highenthalpy flow, two preselected vortex dynamics interactions simulated beforehand by means of a reduced-order model developed by the University of Texas at Arlington research group. The work initially presents the design phase of the pylon injectors based on the prediction capabilities of the aforementioned model, which has been implemented in a computer code, and is capable of calculating the dynamics of finite scale supersonic streamwise vortical structures at a highly reduced computational cost. Later, the results of a set of combustion experiments conducted using hydrogen fuel injected into Mach 2.4 high-enthalpy $(2.8 \mathrm{MJ} / \mathrm{kg})$ airflow are presented and thoroughly discussed. In these experiments, instantaneous OH PLIF measurements at different spanwise stations of the reacting plume downstream of the injection point are used to probe the flow. Although the long-lived nature of hydroxyl radicals implies that their presence in an area of the flow does not necessarily mark the zones in which ignition conditions are achieved, they provide important indications on the plume morphology. $\mathrm{OH}$ radicals, therefore, served as trackers of the flow to mark the plume in this study, showing its evolution under the combined effect of the vortical structures and heat release. In addition, schlieren and $\mathrm{OH}^{*}$ diagnostic techniques have been employed to document the supersonic combustion and flameholding characteristics of the plume. The results show that, for the heat release levels considered in this study, the morphology of the plume and its evolution is very similar to the results produced by the code, enabling an interpretation of the phenomena based on vortex dynamics considerations. The persistence of the streamwise vortical structures created by the selected ramp configurations is shown together with the effectiveness of the coherent structures in successfully anchoring the flame very close to the injection point. The work shows the possibility of a new approach in the design of injection strategies (i.e., not limited to injection devices) suitable for being adopted in scramjet combustors, based on the ability to predict with basic vortex dynamics concepts and a highly reduced computational cost, the main features of flows of technological interest.

\section{Reduced-Order Model for Supersonic Vortex Dynamics}

In this section, a reduced-order model capable of describing the relevant dynamics of an interacting system of streamwise, supersonic, vortical structures is discussed. With this model, the flowfield can be computed once the Mach number of the flow in which the vortices are shed, the convective velocity, the initial relative position of the vortices, and an estimation of the circulation of the large-scale structures are known. A detailed description of the model is provided in [20].

For the high Reynolds numbers typical of the supersonic flows of interest, and the timescales associated with the processes considered here, an inviscid, nondiffusive formulation is considered. Although this simplification will suppress some features of the actual process, it does reproduce, as will be clearly shown later, peculiar features associated with the morphological evolution of the plume of a passive scalar introduced in the vortical flow and subjected to the action of the large-scale stirring structures.

A vortex model consistent with the inviscid description and the assumption that the small-disturbance theory can be applied is the one that follows from supersonic lifting line theory. The streamwise vortices of a supersonic lifting line can influence only the domain defined by the Mach cones that arise from the point of their formation. Closed-form relations for calculating the induced velocity field of the lifting line are obtained by computing the spatial derivatives of the lifting line potential in supersonic flow, which is written in Eq. (1) for trailing vortices lying on the $(x, y)$ plane [21], where $x$ is the streamwise direction as shown in the schematic in Fig. 1a:

$$
\Phi=\frac{\Gamma}{2 \pi}\left[\tan ^{-1} \frac{x(y-1)}{z \sqrt{\Omega}}-\tan ^{-1} \frac{x(y+1)}{z \sqrt{\Omega}}\right]
$$

where

$$
\Omega=x^{2}-\left(M^{2}-1\right)\left[(y-1)^{2}+z^{2}\right]
$$

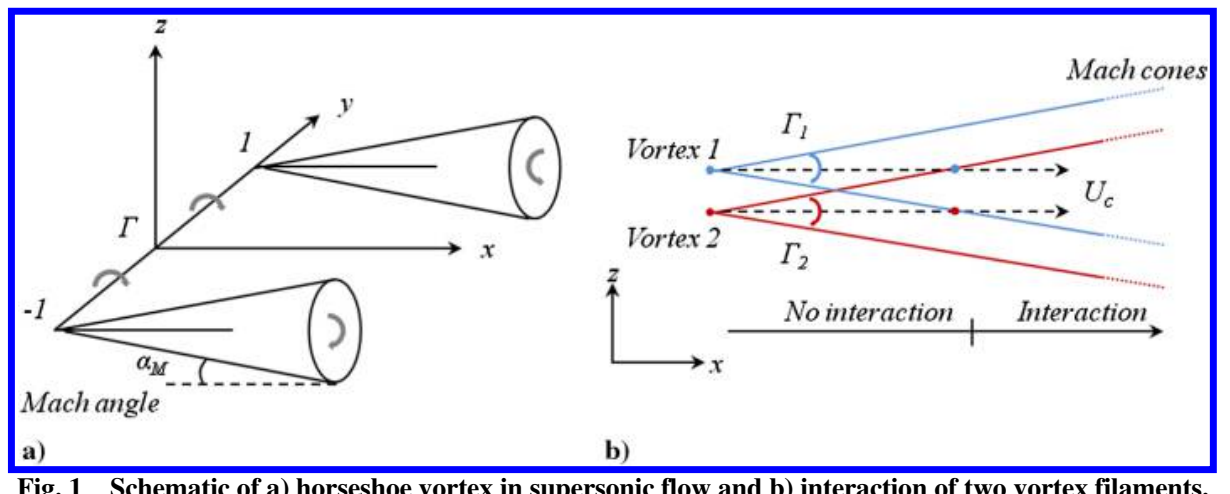

Fig. 1 Schematic of a) horseshoe vortex in supersonic flow and b) interaction of two vortex filaments. 
The surface represented by $\Omega=0$ defines the Mach cone surrounding the streamwise vortex filaments and is also the boundary of influence of each filament. The potential is other than zero only within the Mach cones, whereas it is zero in the remaining portion of the space. The two velocity components $v(x, y, z)$ and $w(x, y, z)$, representing the flow in a plane perpendicular to the streamwise vortex in a single generic Mach cone of a counterclockwise-spinning vortex evolving along the $x$ axis and starting at $\left(0, y_{s}, z_{s}\right)$, are shown in Eqs. (3) and (4):

$$
\begin{gathered}
v(x, y, z)=\frac{\Gamma}{2 \pi} \frac{x\left(z-z_{s}\right)}{\left[\left(z-z_{s}\right)^{2}+\left(y-y_{s}\right)^{2}\right] \sqrt{\Omega}} \\
w(x, y, z)=-\frac{\Gamma}{2 \pi} \frac{x\left(y-y_{s}\right)\left[\Omega-\left(M^{2}-1\right)\left(z-z_{s}\right)^{2}\right]}{\left[\left(z-z_{s}\right)^{2} \Omega+\left(y-y_{s}\right)^{2} x^{2}\right] \sqrt{\Omega}}
\end{gathered}
$$

These velocity components become infinite at the cone's surface and tend to the induced velocities obtained by a vortex filament in incompressible flow (i.e., the Biot-Savart law) for values of the downstream coordinate $x$ that tend to infinity. When several vortices are considered, the $i$ th vortex induces a velocity $\overline{\boldsymbol{V}}_{i}=\left(v_{i}, w_{i}\right)$ only in the portion of the $(y, z)$ plane enclosed within the boundaries of its Mach cone. As a consequence, it is not possible for two (or more) vortical structures to interact when they are outside of their respective Mach cones (see Fig. 1b). The mutually induced velocities on each vortex can be calculated as the superposition of the effects of the other $N-1$ vortices in the system

$$
\overline{\boldsymbol{V}}_{i}=\sum_{j=1}^{N-1} \overline{\boldsymbol{V}}_{j}
$$

with $i \neq j$ accounting for their relative position, circulation, and Mach cone aperture. To obtain a closed system of equations for the translational motion of the vortices, Helmholtz's result that the vortex lines are material lines is used. Hence, the point vortex equation on the unbounded plane for the generic vortex $i$ th can be written as $\mathrm{d} \bar{r}_{i} / \mathrm{d} t=\overline{\boldsymbol{V}}_{i}$, where $\bar{r}_{i}$ is the position vector. The singularity of the point vortex is removed by assigning a finite size core to the vortex filaments. Large-scale vortices are constructed by means of a given initial circulation distribution of a number of vortex filaments arranged in a coherent structure of a chosen dimension. A Gaussian distribution of vorticity is used to resemble that of a Burgers vortex $[22,23]$.

\section{Experiment Design}

The reduced-order model developed by the University of Texas at Arlington group [20] has been used to produce the simulation of two distinct vortical systems. In the first case, a symmetric disposition of the vortices in the flowfield was chosen; the second case was meant to emphasize the effect of the dynamics of the vortical structures in an asymmetric configuration.

Nonreacting experiments in supersonic flow have already shown the intriguing possibility of capturing peculiar flow physics with a certain degree of accuracy by means of the aforementioned code [15]. The same methodologies have been used for the design of the experiments that were to be conducted in the expansion tube facility of Stanford University.

In the preliminary experiment design, it was decided that the bodies of both injectors should have been a pylon design $38 \mathrm{~mm}$ in height (more details are provided in Sec. IV.B): the fins were to be enclosed within the inviscid core of the flow generated in the expansion tube. Expansion ramps of varying geometry and relative displacement were added to the basic pylon geometry in two configurations, which were designed to target the vortex dynamics of interest as predicted by the reduced-order code. Injection took place along the slit located at the base of the ramps, spanning nearly the entire height of the pylons. Figure 2 shows a CAD model of the expansion tube's test section and the inviscid core region on the flat plate assembly designed for the experiment. The longitudinal extent of the inviscid core of the expansion tube's freejet was approximately $15 \mathrm{~cm}$.

In the simulations, the initial position of the cores of the vortices in the CVP forming downstream of the ramps was set at two-thirds of the total ramp height to account for downwash, which was found to be a good approximation during the cold flow studies previously conducted $[14,15]$. The vertical distances of the vortices were made to coincide with the width of the ramps in use, the extent of which was chosen after a set of simulations was performed to find configurations that resulted in peculiar flow physics. Although the total amount of vorticity constituting the vortices is fixed in the flow due to the inviscid nature of the calculations, the shape of the vortex patches can evolve due to the velocity field imposed by the other structures to fulfill the Helmholtz theorem stating that vortex lines are material lines convecting with the local fluid velocity. The conditions used to reproduce the actual flow in the expansion tube are freestream Mach number of 2.4, a corresponding convective velocity of the structures of $1700 \mathrm{~m} / \mathrm{s}$, and maximum length of the probed area equal to $10.7 \mathrm{~cm}$. As can be inferred by the convective velocity, the residence time of the flow is on the order of $10^{-5} \mathrm{~s}$, therefore, it was crucial to choose a value of the circulation of the vortices that would generate measurable modifications in the flowfield within the limited test area. Based on the relation available from Waitz et al. [8], relating the geometry of the ramp to the circulation based on the freestream flow condition, it was possible to size the expansion ramps to be capable of introducing streamwise vortices with a strength of approximately $1.2 \mathrm{~m}^{2} / \mathrm{s}$. A diameter of $2 \mathrm{~mm}$ was assigned to the viscous core of each patch of vorticity because it was expected to scale with the chosen height of the ramps $(2.8 \mathrm{~mm})$; the distribution of vorticity across the rotational core has been assumed Gaussian based on previous findings available in literature [22,23]. Four cross-sectional planes have been considered, specifically, $1.8,4.3,7.6$, and $10.7 \mathrm{~cm}$

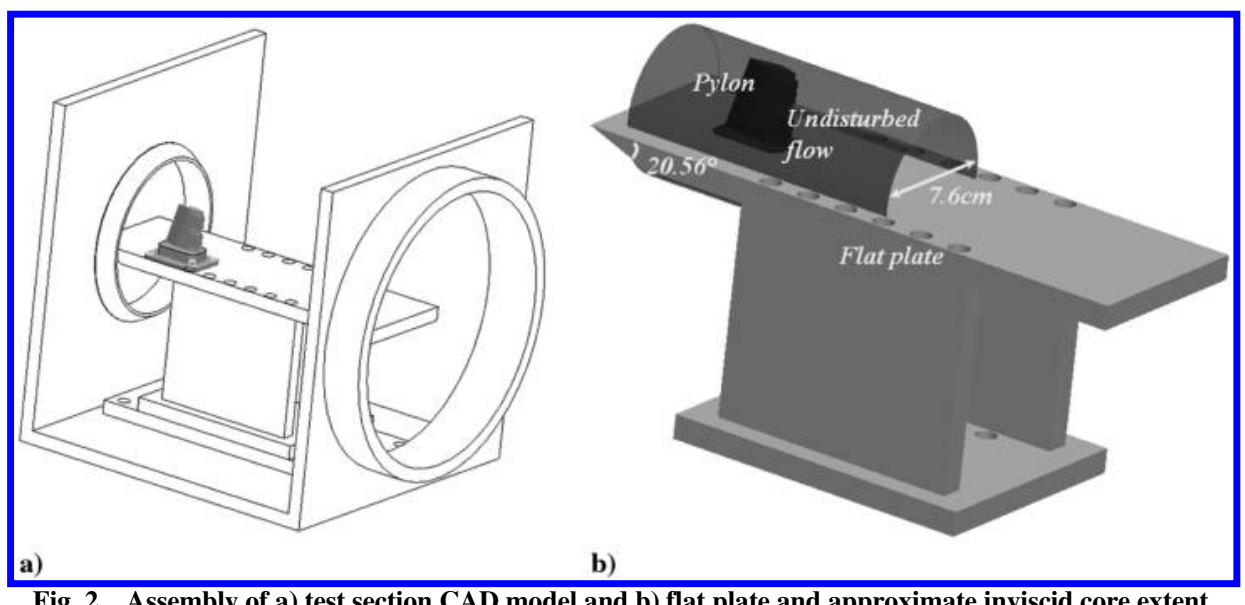

Fig. 2 Assembly of a) test section CAD model and b) flat plate and approximate inviscid core extent. 




Fig. 3 Evolutionary image of the streamwise vortices: symmetric case.

downstream of the injection point. The results presented are those that reproduce the actual displacement of the ramps chosen for the final injector concepts designed afterward. Although the code in use does not account for the diffusion of reactants in the vortical structures, a qualitative study of the shape of the plume was possible by simulating the kinematic transport of virtual tracers, which have the function of marking the simulated trajectory of a fuel exiting from the injection slit. This was essential to produce comparisons with the experimental results because the available instrumentation for the experimental portion of the study permitted the visualization of the reacting plume of the injectors through the use of OH PLIF, but would not be able to provide any information on the velocity flowfield.

As already discussed, two separate pylon configurations were investigated both numerically and experimentally. The first case was chosen as a base case, in which four ramps were placed symmetrically about the $z$ axis, resulting in four symmetrically oriented CVPs. Figure 3 shows the three-dimensional evolutionary pattern of the expected vorticity patches of the streamwise vortices, whereas Fig. 4 shows the cross-sectional vorticity contours and the snapshots of a simulated injectant (i.e., the virtual particles) taken at the same four positions that were chosen for the experimental campaign. The three-dimensional plot of the vorticity evolution was created using the lambda-2 criterion [24] to recognize and mark the surfaces of vortical structures, whereas the fill-color indicates the vorticity along each surface. Because of the reduced-order nature of the analytical model used in the code, the simulated flowfields must be considered the time-averaged flowfields expected of the experimental case. As can be inferred from the trajectories of the vortex filaments in Fig. 3, the first and second stations (Figs. 4a and $4 \mathrm{~b}$ ) did not present noticeable changes in the position of the cores. On the other hand, a sudden change in the relative positions of the vortices from the 4.3 to the $7.6 \mathrm{~cm}$ station (Fig. 4c) was present. The positions of the cores do not seem to change noticeably from 7.6 to $10.7 \mathrm{~cm}$ (Fig. 4d). An interesting feature of the symmetric configuration was the attitude of the quadruplet of vortices at the center of each image: As the vortices interacted and moved downstream, the quadruplet separated in two vortex pairs, approaching the CVP systems on the top and the bottom, respectively. It is worth noting that the mutual distances of the vortex cores along the $y$ axis remained nearly unchanged (approximately $5 \mathrm{~mm}$ ), while becoming shorter in the $z$ direction.

Figures 5 and 6 show the evolution of the vortical structures and the details of the cross sections for the asymmetric ramp configuration as predicted by the code. For this configuration, only three stations were surveyed in the experimental campaign, and these stations are presented in Fig. 6 for the numerical investigation. Figure 5 indicates

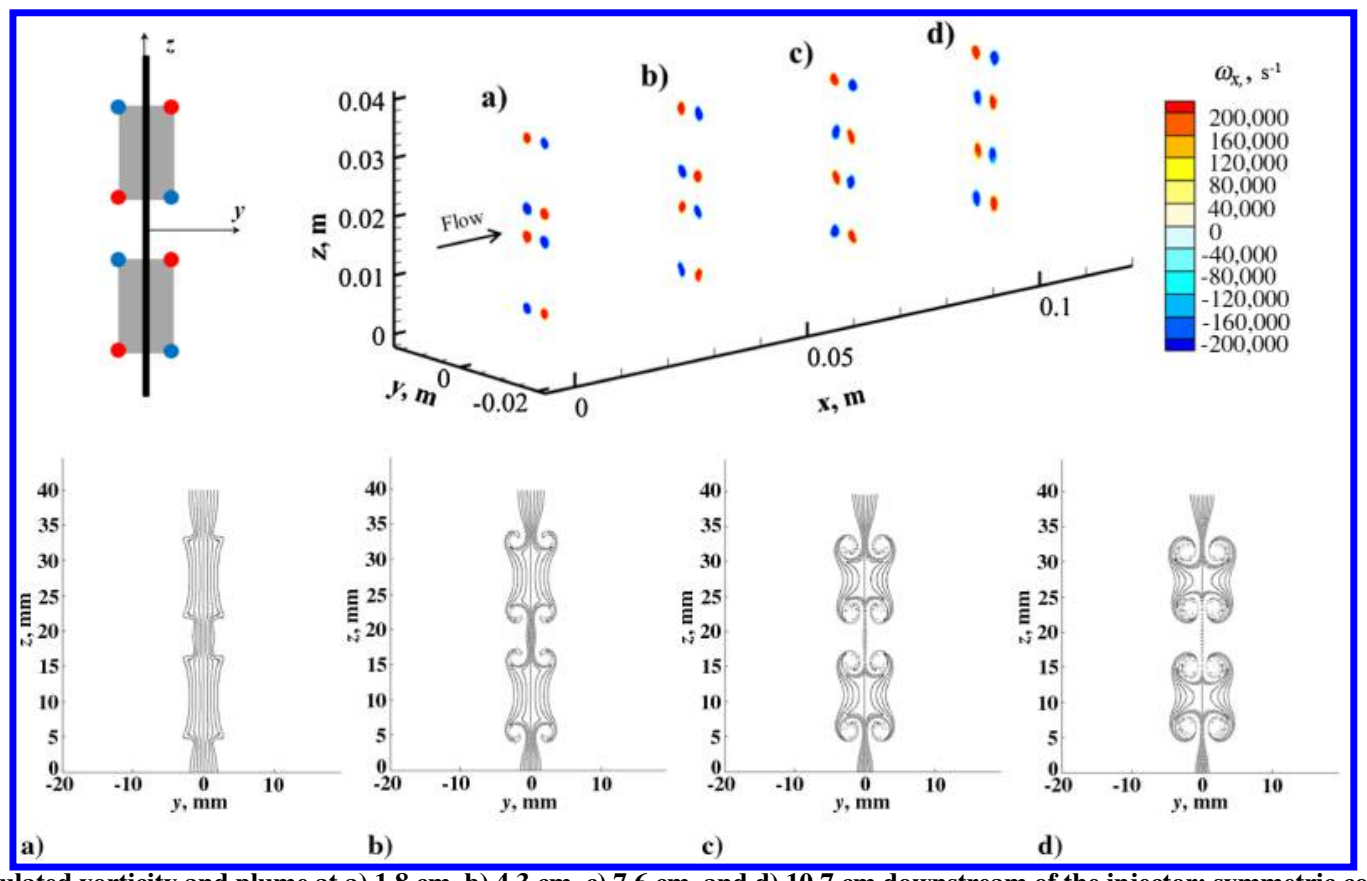

Fig. 4 Simulated vorticity and plume at a) $1.8 \mathrm{~cm}$, b) $4.3 \mathrm{~cm}, \mathrm{c}) 7.6 \mathrm{~cm}$, and d) $10.7 \mathrm{~cm}$ downstream of the injector: symmetric configuration. 




Fig. 5 Evolutionary image of the streamwise vortices: asymmetric case.

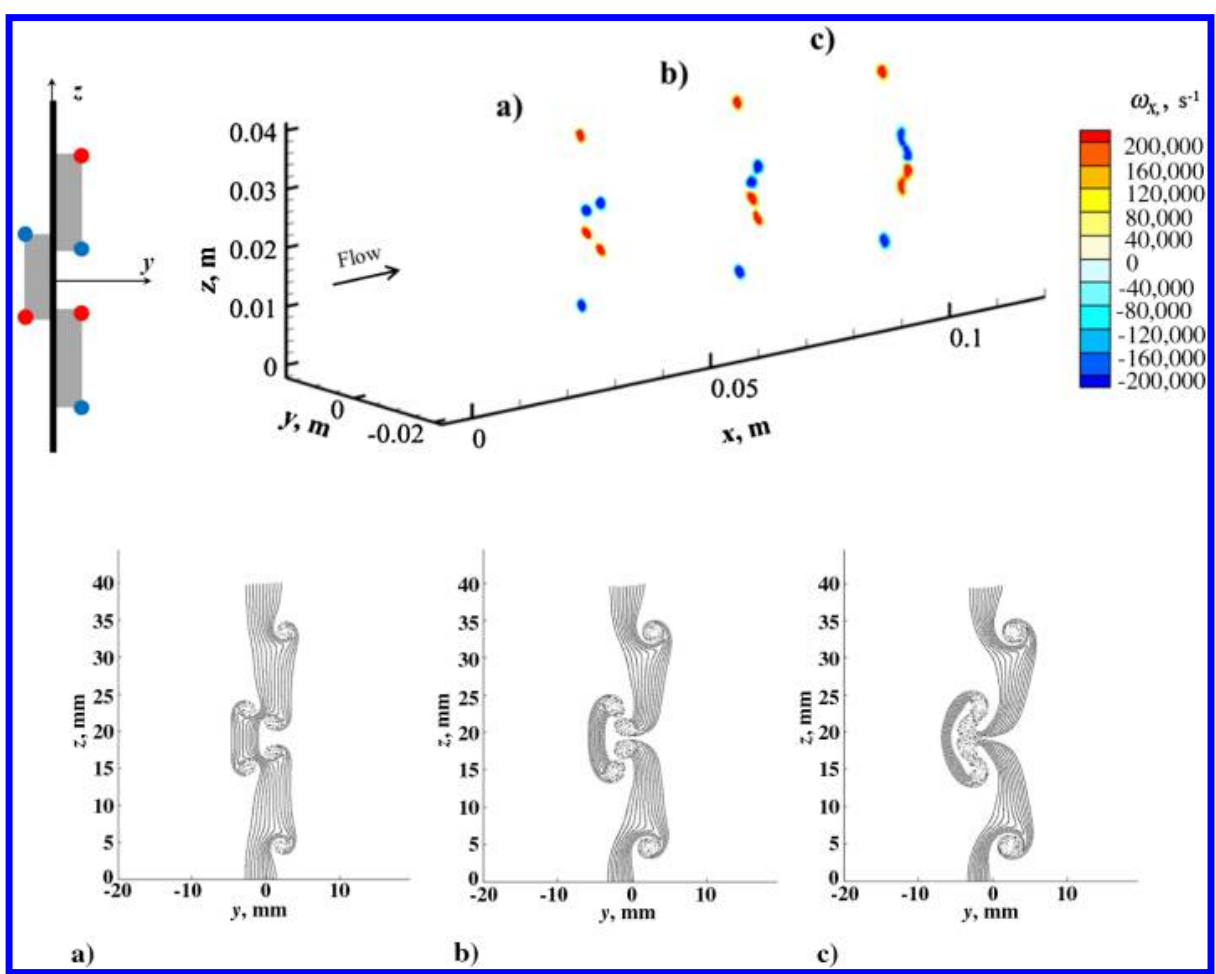

Fig. 6 Simulated vorticity and plume at a) $1.8 \mathrm{~cm}$, b) $4.3 \mathrm{~cm}, \mathrm{c}) 7.6 \mathrm{~cm}$, and d) $10.7 \mathrm{~cm}$ downstream of the injector: asymmetric configuration.

that both single vortices in proximity of the wall and on the top kept a nearly straight trajectory; this suggests that they were not greatly affected by the flowfield evolving at the center of the system maintaining their initial positions on the $(y, z)$ plane. The detailed cross-sectional planes in Fig. $\underline{6}$ clearly show that the two internal CVPs shed by the two wider ramps on the opposite side of the injector acted exactly as an independent CVP. The vortex pair moved toward the negative values of the $y$ axis due to its autoinduced velocity field, whereas the CVP generated from the small ramp created a favorable velocity field to accelerate their movement toward the left, promoting the merging of the corotating structures.

The numerical simulation of the plume expected at the four cross-sectional planes for both the symmetric and asymmetric configurations at $10.7 \mathrm{~cm}$ is presented in Figs. 7a and $7 \mathrm{~b}$, respectively. In the symmetric configuration, the agglomeration of vortices in the two areas was expected to create a lobed plume, with two clear bulges located along the $z$ axis. The simulated plume for the asymmetric case shows a remarkable difference compared with the symmetric configuration: The wide lobe at the center of the image is generated by the two pairs of merging corotating vortices, whereas the smaller lobes at the extremities exist due to the effect of the single vortices.
The channel created by the central vortical system can be seen in the cusp generated at the center of the plume.

\section{Experimental Setup and Test Matrix}

\section{A. Test Facility}

All tests were conducted at the expansion tube facility of Stanford University. An expansion tube is an impulse flow facility that generates high-enthalpy flows and can be used to reproduce conditions relevant to hypersonic flight and supersonic combustion. The expansion tube is composed of type 304 stainless-steel circular sections (outer diameter, $16.5 \mathrm{~cm}$; inner diameter, $14 \mathrm{~cm}$ ): a driver section ( $1.9 \mathrm{~m}$ long), a driven section (7.3 $\mathrm{m}$ long), and an expansion section ( $2.8 \mathrm{~m}$ long). Test gas conditions are generated by the unsteady expansion of the shock-treated driven gas; the test gas subsequently flows through a $30 \times 30 \times 30 \mathrm{~cm}^{3}$ test section and exhausts into a $0.6 \mathrm{~m}^{3}$ dump tank. The test section has optical access on three sides. The final test gas conditions (i.e., Mach, temperature, and pressure) can be adjusted by changing the filling pressure of each section. For a more thorough description of the facility and its operation, see Heltsley et al. [25]. 


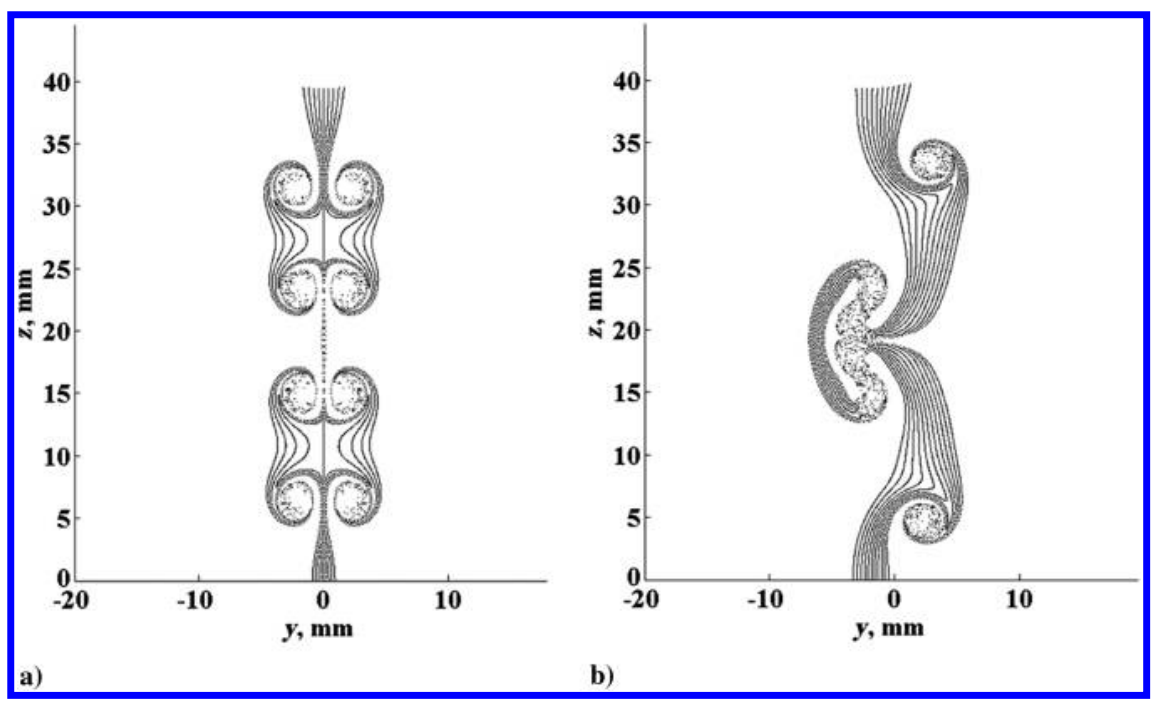

Fig. 7 Simulated injection plume at $10.7 \mathrm{~cm}$ downstream of the injection point: a) symmetric case; b) asymmetric case.

The conditions chosen for this work were Mach number $M=2.4$, static temperature $T=1400 \mathrm{~K}$, and static pressure $p=40 \mathrm{kPa}$. The stagnation enthalpy was $2.8 \mathrm{MJ} / \mathrm{kg}$. These conditions approximately correspond to Mach 8 flight at $30 \mathrm{~km}$ altitude. The test time was approximately $500 \mu \mathrm{s}$. The uniform flow downstream of the exit plane of the expansion tube is confined by a region of 7.6-cm-diam core bounded by the limiting characteristic lines of the incoming flow, and it extends approximately $15.2 \mathrm{~cm}$ downstream of the tube exit. The pylon injectors were mounted on a flat plate. Because of the limited length of the undisturbed flow region, the leading edge of the flat plate was located as far upstream as possible (approximately $4.1 \mathrm{~cm}$ into the expansion section), thus preventing the reflection of shocks generated by the model from impinging on the expansion tube wall, while keeping the entire fin in the field of view. The test section assembly is that shown in Fig. 2. The flat plate was not equipped with any ignition or flameholding devices, which are well known to promote these phenomena [26].

\section{B. Design of the Injectors}

Hypermixers for supersonic flow have been widely studied in recent years. Some of these works have been employed to generate a database adopted as a reference for the proper sizing of the body of the injectors so that it could be considered in line with the latest investigated configurations. Indications on the possible geometry were taken from Hsu et al. [17] who tested different injection fins with slenderness ratios (i.e., the ratio between the fin thickness and their length at the root) ranging from 0.16 to 0.24 , and Doster et al. [18], who used in-stream pylons with slenderness ratio of 0.16 . The final design of the pylon injectors is characterized by a slenderness ratio of 0.19 , resulting in an injection configuration which is comparable with recent studies. Because the main focus of the work was the survey of the reacting plume evolution in the presence of selected streamwise vortex configurations, the injectors were carefully sized to serve as platforms to be equipped with four and three expansion ramps respectively. The two pylon injectors are depicted in Fig. 8; in Fig. 9 the detailed design of the symmetric ramp configuration is shown.

The body of the injectors was designed with a diamond-like planview cross section, swept downstream at $15 \mathrm{deg}$. The ramps were positioned on both sides of the pylons. The fuel was delivered by a thin slit located along the centerline of the trailing edge, which spanned the length between the two outside edges of the ramps with an outlet area of $15.5 \mathrm{~mm}^{2}$. The slit height was $30 \mathrm{~mm}$. The height $h$ of each ramp was $2.8 \mathrm{~mm}$ and the expansion angle $\alpha_{e}$ was $15 \mathrm{deg}$. The final outcome of the simulations presented in Sec. III was the location and geometry of the ramps to be used on the pylons. For the symmetric case, the ramps were chosen to be $W=12 \mathrm{~mm}$ wide and $5.1 \mathrm{~mm}$ apart. As far as the asymmetric configuration, two ramps ( $W=12 \mathrm{~mm}$ ) were chosen to be mounted on one side of the pylon and a 7-mm-wide, smaller ramp in the center on the opposite side.
The injectors were rapid prototyped using a high-performance resin, which proved robust enough to complete the test matrix despite the harsh conditions in the expansion tube. The maximum injection plenum pressure needed to be within the structural limits imposed by the material selection; for this reason, experiments were conducted with relatively low values of jet-to-freestream momentum flux ratios. Hydrogen was delivered to the injector by a fast-acting solenoid valve.

\section{Schlieren Photography}

A Z-type schlieren system was used to instantaneously image the flowfield downstream of the pylons. A xenon spark lamp was used to illuminate the test section, and a LaVision Imager Intense chargecoupled device (CCD) camera was used to acquire images with a 500 ns exposure. Background and dark noise subtraction was performed on all acquired schlieren images.

\section{OH* and $\mathrm{OH}$ Planar Laser-Induced Fluorescence}

$\mathrm{OH}^{*}$ chemiluminescence was used to ascertain global features of the reacting plume because its signal has been proved to be indicative of heat release rates [27,28]. An Andor iStar intensified CCD (ICCD) camera fitted with a $100 \mathrm{~mm}, f 2.8 \mathrm{UV}$ lens, was used in conjunction with a $10 \mathrm{~nm}$ full width at half-maximum interference filter centered at $313 \mathrm{~nm}$ to capture chemiluminescence from the chemically excited $\mathrm{OH}$, which is used as a tracer for areas of the flow undergoing combustion. Tests were conducted using air as the test medium and hydrogen as the injectant $\left(\mathrm{H}_{2}\right.$ /air operation). A preliminary test using oxygen instead of air was conducted to assess the viability of the intended configuration with respect to spontaneous ignition and flameholding characteristics. Different integration times (i.e., gates) were used for each case (50 $\mu$ s for $\mathrm{H}_{2} / \mathrm{O}_{2}$ and $100 \mu$ s for $\mathrm{H}_{2} /$ air).

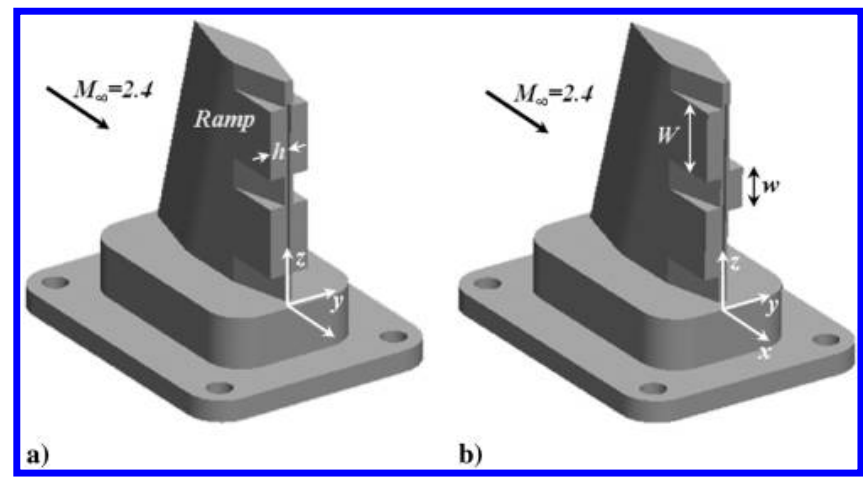

Fig. 8 CAD model of the two pylon injector configurations used in the experiments. 


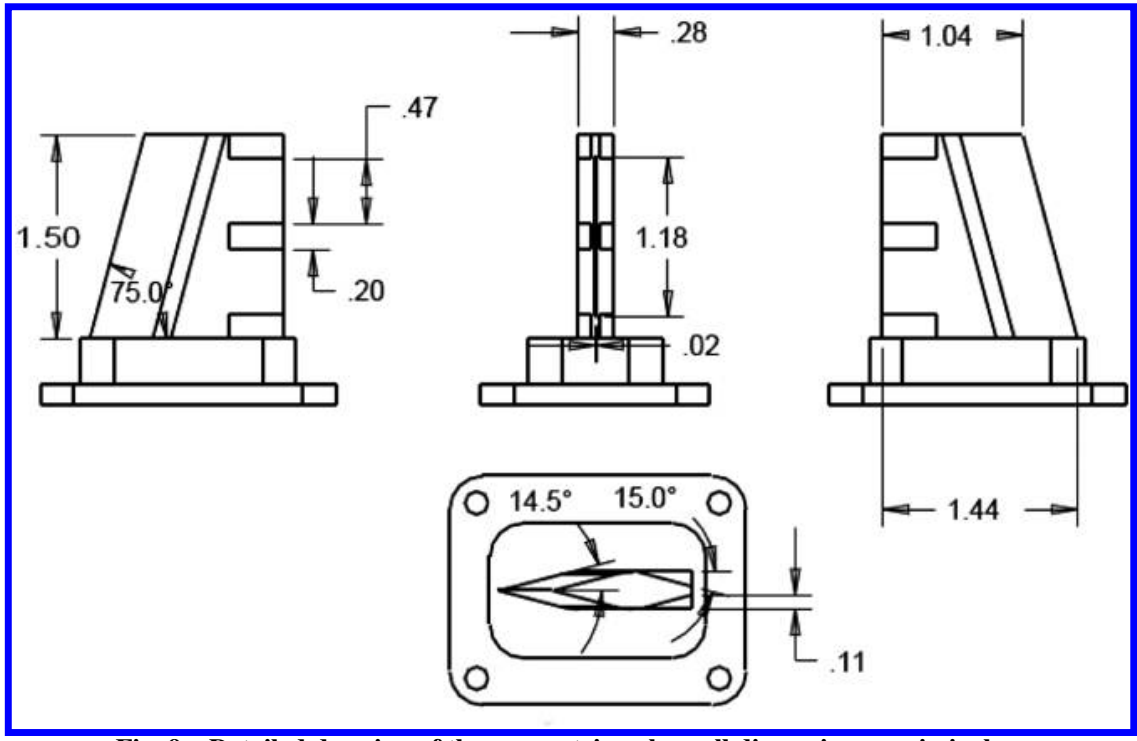

Fig. 9 Detailed drawing of the symmetric pylon; all dimensions are in inches.

Background subtraction was performed. Results of the $\mathrm{OH}^{*}$ imaging were used to define the location of the streamwise sections to be inspected with OH PLIF imaging.

The same ICCD camera (Andor iStar 712) and interference filter were used to acquire OH PLIF images. A frequency doubled neodymium yttrium aluminum garnet at $532 \mathrm{~nm}$ pumped a Lumonics HD-500 dye laser to produce $566.6 \mathrm{~nm}$ light, which was frequency doubled to $283.3 \mathrm{~nm}$, corresponding to the $Q 1(n=7) A-X(1,0)$ transition of $\mathrm{OH}$. This transition results in a signal that is nearly proportional to the $\mathrm{OH}$ mole fraction and is weakly dependent on pressure and temperature, providing instantaneous information regarding the location of reaction zones. Cylindrical lenses were used to form a light sheet, which illuminated the planes normal to the freestream flow direction located 1.8, 4.3, 7.6, and $10.7 \mathrm{~cm}$ downstream of the fuel injection plane. The ICCD camera was gated for $100 \mathrm{~ns}$. The camera was positioned at an angle relative to the test section (Fig. 10b), and a grid was imaged at each object plane to allow for remapping of the image onto Cartesian coordinates. A $100 \mathrm{~mm}$, $f 2.8 \mathrm{UV}$ lens was used for the imaging. Depending upon the particular conditions being imaged, the lens was stopped up or down from $f 2.8$ to $f 5.6$ to avoid saturation; images were then scaled to approximately account for the variation of captured light intensity. Background subtraction and correction for laser sheet nonuniformity were also performed on all acquired images. Shot-to-shot variation in incident laser energy was tracked using a photodiode, and images were corrected for these fluctuations as well. Following this correction approach, all OH PLIF image data results are expected to be an approximate representation of the relative $\mathrm{OH}$ distribution in different regions.

\section{E. Test Matrix}

Tests were performed in air and oxygen freestream flows with two injection stagnation pressures. From the stagnation pressure, the density and the velocity of the fuel can be computed; consequently, the jet-to-freestream momentum flux ratio can then be calculated using Eq. (5):

$$
\bar{q}=\frac{\gamma_{j} p_{j} M_{j}^{2}}{\gamma_{\infty} p_{\infty} M_{\infty}^{2}}
$$

Hydrogen was injected with a total temperature of $300 \mathrm{~K}$ and two different stagnation pressures, depending on the experiment. The chosen injection stagnation pressures were $p_{0, j 1}=43 \mathrm{kPa}$ and $p_{0, j 2}=65 \mathrm{kPa}$, corresponding to injection Mach numbers of 0.34 and 0.87 . The specific heat ratio of the freestream was approximately 1.3 , resulting in jet-to-freestream momentum flux ratios of 0.02 and 0.14 and mass flow rates of 0.22 and $0.56 \mathrm{~g} / \mathrm{s}$, respectively. The complete test matrix is shown in Table 1.

A pressure transducer (PCB Piez̄otronics, model 112A22) mounted downstream of the solenoid valve enabled time measurements of the injector stagnation pressure. The plenum pressure of the injector was nearly constant for the entire duration of each test, as shown in Fig. 11. The $1 \mathrm{~ms}$ window shown in Fig. 11 includes the $500 \mu \mathrm{s}$ of test time, which extends from approximately 4.75 to $5.25 \mathrm{~ms}$ (see the dashed lines). The two constant values of pressure represent the absolute total pressures obtained in the injector plenum for the two conditions considered in the study.

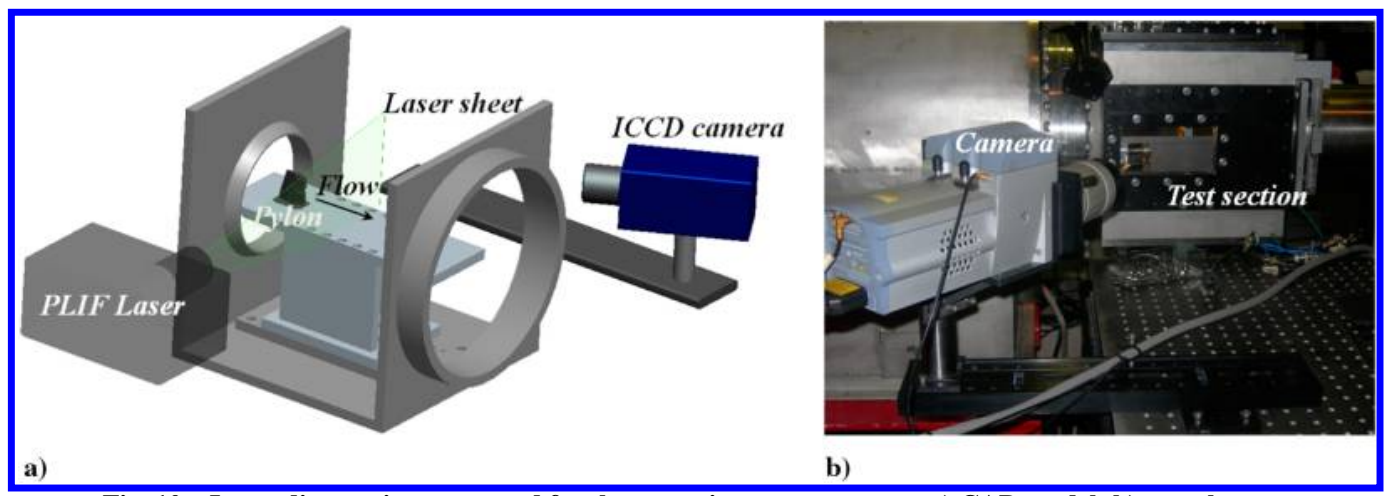

Fig. 10 Laser diagnostics setup used for the spanwise measurements: a) CAD model; b) actual setup. 
Table 1 Test matrix

\begin{tabular}{|c|c|c|c|c|c|c|c|c|}
\hline Configuration & Schlieren & $\mathrm{OH}^{*}$ & OH PLIF & Oxidizer & $M_{\infty}$ & $T_{\infty}, \mathrm{K}$ & $p_{0, j}, \mathrm{kPa}$ & $\dot{m}_{i}, \mathrm{~g} / \mathrm{s}$ \\
\hline Symmetric & a & -- & -- & air & 0 & 300 & 43 & 0.22 \\
\hline Symmetric & a & -- & a & air & 2.4 & 1400 & 43 & 0.22 \\
\hline Symmetric & a & -- & -- & $\mathrm{O}_{2}$ & 0 & 300 & 65 & 0.56 \\
\hline Symmetric & a & a & -- & $\mathrm{O}_{2}$ & 2.4 & 1400 & 65 & 0.56 \\
\hline Symmetric & a & a & a & air & 2.4 & 1400 & 65 & 0.56 \\
\hline Asymmetric & -- & -- & a & air & 2.4 & 1400 & 43 & 0.22 \\
\hline Asymmetric & -- & -- & a & air & 2.4 & 1400 & 65 & 0.56 \\
\hline
\end{tabular}

Tests that have been conducted.

\section{Experimental Results}

\section{A. Schlieren Photography: Symmetric Configuration}

Schlieren images of the flow due to injection into quiescent air have been taken for both injection stagnation pressures (Fig. 12) with the symmetric pylon. For these experiments, the pressure in the test section was set to $40 \mathrm{kPa}$ to reproduce the static pressure of the flow during the run. These images were necessary to assess the uniformity and the characteristics of the injection process. The field of view shown in the images is $4 \times 13 \mathrm{~cm}^{2}$. In all the images discussed next, the $x$ axis origin refers to the injection point.

The images document a uniform fuel distribution along the vertical slit. This is an important verification that excludes the possibility of interference effects, on a vortex dynamics standpoint, due to vortical structures unintentionally introduced by a distortion of the injectant during the injection process. Because the internal design of the plenum of both pylons was the same, these results were also considered valid for the asymmetric configuration.

Figure 13 displays schlieren image data for both injection stagnation pressures during a reactive test with the symmetric pylon. Shocks can be recognized in both images downstream of the injection point up to $x=40 \mathrm{~mm}$. Because the injection was subsonic, the shocks were attributed to the complex flow structure generating at the base of the pylon. Despite the images showing two-dimensional pathintegrated results, a careful inspection of Fig. $13 \mathrm{~b}$ shows an important flow characteristic: ranging from $x=30 \mathrm{~mm}$ to the end of the probed area, a zone of large density gradients can be seen; the area is delimited by a dashed line in the image. These gradients can be generated by a combined effect of the three-dimensional development of the streamwise vortices in the plume and the local heat release due to the turbulent combustion. This consideration is corroborated by the $\mathrm{OH}^{*}$ surveys described in Sec. V.B.

\section{B. OH* Imaging: Symmetric Configuration}

$\mathrm{OH}^{*}$ chemiluminescence images were acquired for air and oxygen freestreams at the highest injection stagnation pressure (see Table 1) to gain an insight into the global time-averaged structure of the reactive plume issuing from the symmetric pylon. A test in pure

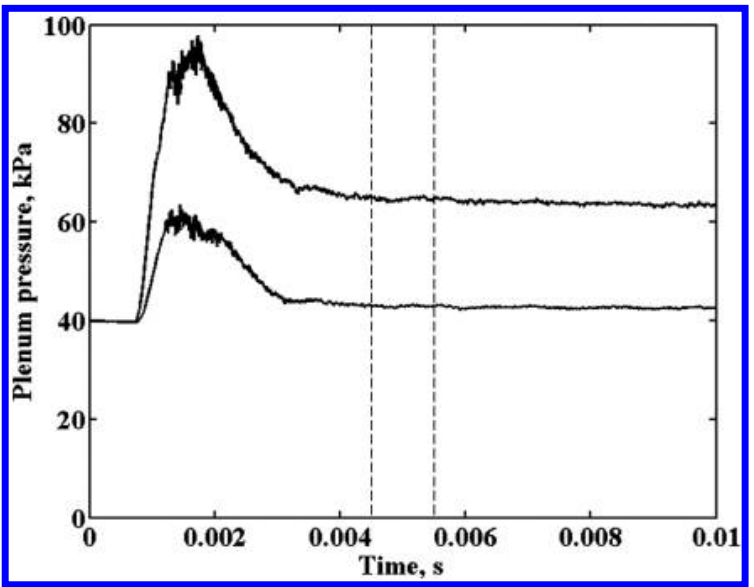

Fig. 11 Typical time history of the plenum pressure in the injector vessel. oxygen was carried out to assess if spontaneous ignition could have been effectively achieved. Figures $14 \mathrm{a}$ and $14 \mathrm{~b}$ show the tests conducted with $\mathrm{O}_{2}$ and air as freestream flows, $\overline{\text { respectively. }}$

Figure 14a shows the results obtained in the case of $\mathrm{H}_{2} / \mathrm{O}_{2}$ combustion. Two distinct symmetric chemiluminescent regions can be recognized. Both luminescent zones bent toward the centerline of the jet symmetrically. Two confined areas characterized by a

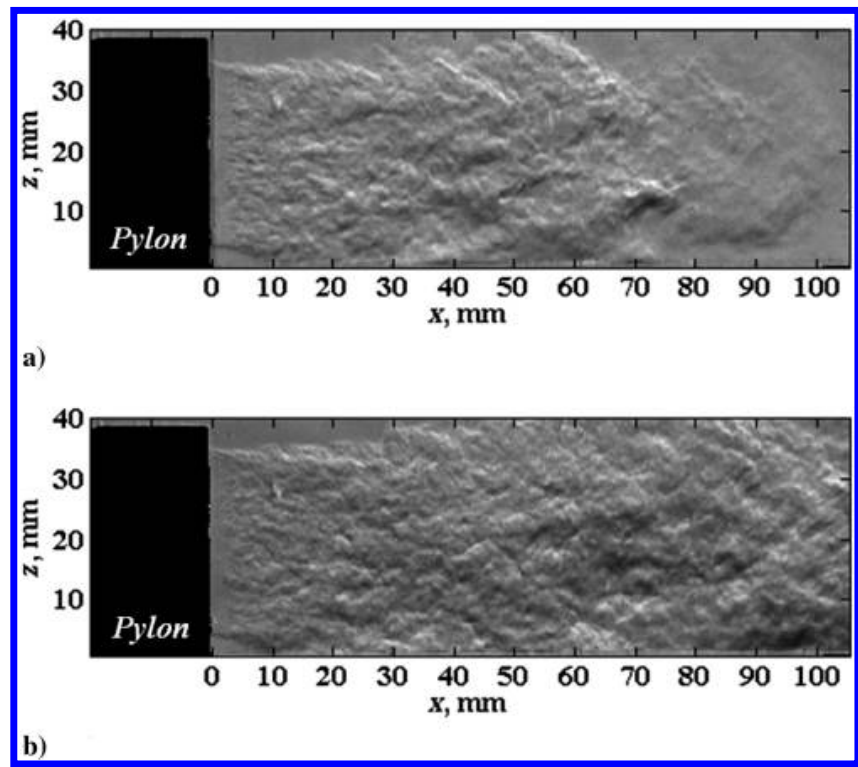

Fig. 12 Instantaneous schlieren images of the quiescent hydrogen jet: a) $p_{0, j 1}=43 \mathrm{kPa}$; b) $p_{0, j 2}=65 \mathrm{kPa}$.

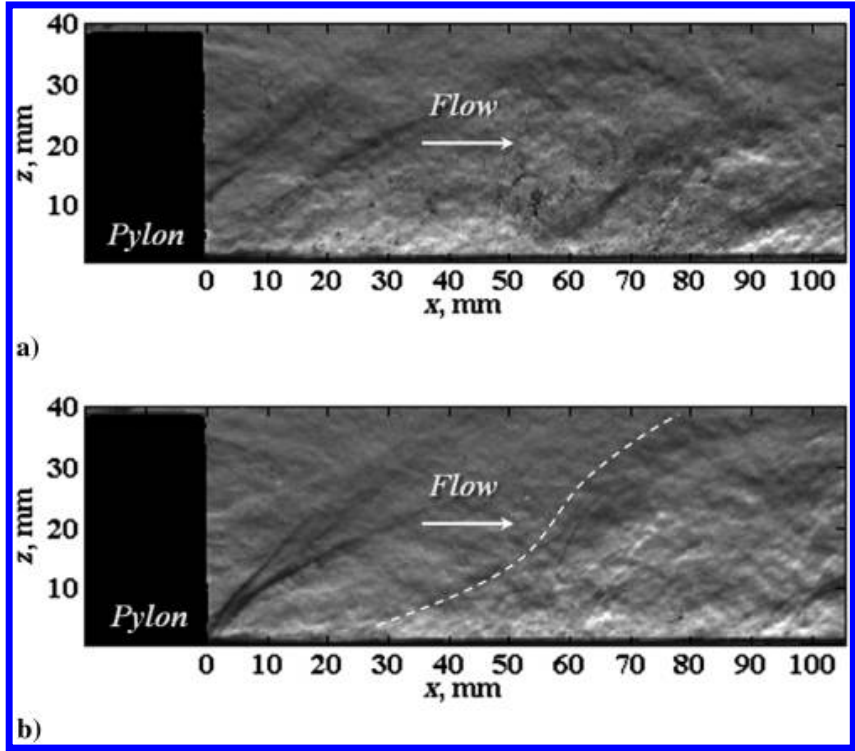

Fig. 13 Instantaneous schlieren images of the runs: a) $p_{0, j 1}=43 \mathrm{kPa}$; b) $p_{0, j 2}=65 \mathrm{kPa}$. 


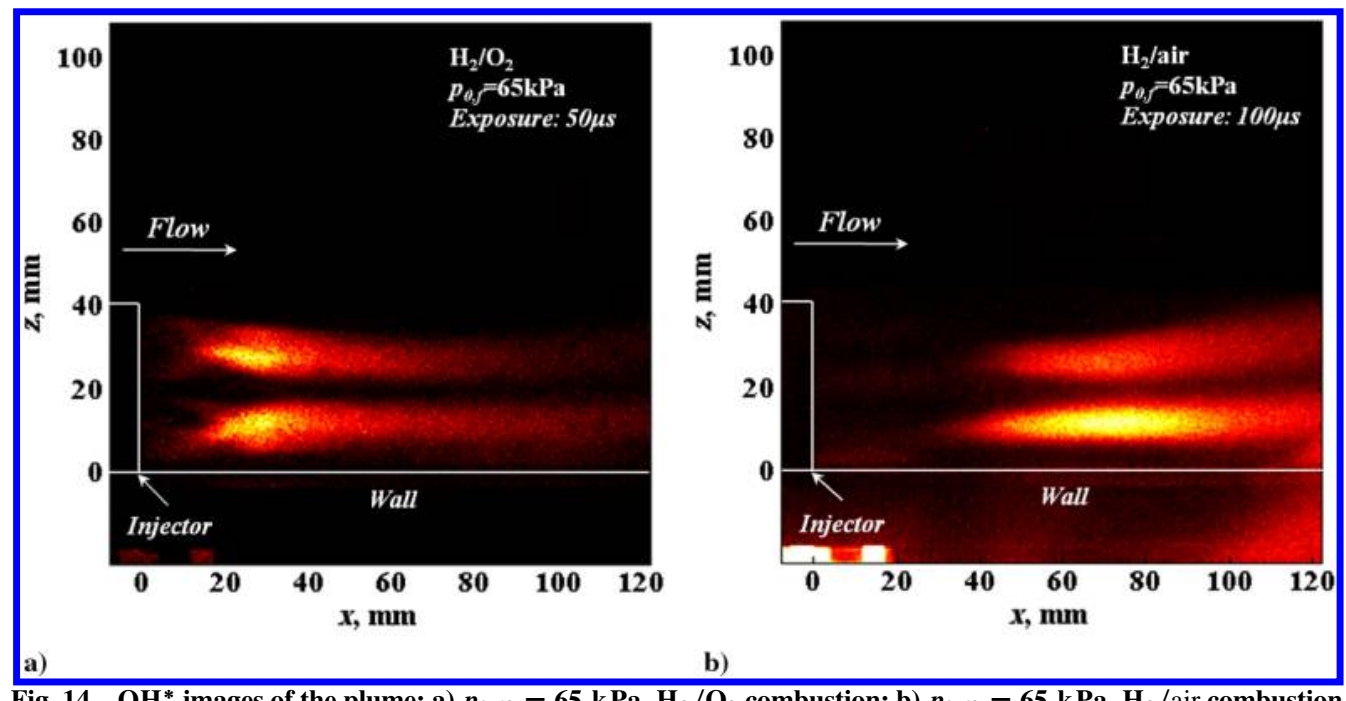

luminescence peak were recognized close to the injection point, between $x=20$ and $40 \mathrm{~mm}$. Because the increase in the chemiluminescence signal is indicative of heat release rates, its weakening in the far field (from $x=50$ to $120 \mathrm{~mm}$ ) can indicate either a consumption of the fuel or flame quenching.

Figure $14 \mathrm{~b}$ shows the $\mathrm{H}_{2}$ /air reacting streamwise plume. Two elongated chemiluminescent regions were present here as well, but in this case, the areas were characterized by a different pattern of luminescence. Ignition took place later compared with the $\mathrm{O}_{2}$ freestream, approximately $30 \mathrm{~mm}$ downstream of the injection point. Higher heat release rates can be inferred in the bright region closer to the wall (between $x=40$ and $100 \mathrm{~mm}$ ) suggesting a faster combustion that, due to its location, could have been affected by the presence of a turbulent boundary layer. The effect of the plume bent upward in the far field could be attributed either to the termination of the perfectly undisturbed flow characteristics of the expansion tube or the dilatation of the vortical structures due to heat release. Interestingly, the limits of the ignition zone resemble the shape of the region characterized by large density gradients highlighted by the schlieren imaging, as described in Sec. V.A (see Fig. 13b).

Although a direct comparison between the images cannot be conducted due to the difference in integration times used, the presence of two distinct chemiluminescent regions is clear and suggests that molecular mixing and reacting zones were affected and shaped by the streamwise vortical structures created by the symmetric injector. This behavior was also confirmed by the spanwise OH PLIF measurements (see Sec. V.C).

\section{OH PLIF: Symmetric Configuration}

The images in Fig. 15 show the evolution of the reacting plume at different stations downstream of the injection point. The detailed PLIF images for both injection conditions are shown in Fig. 16 at four spanwise stations: $1.8,4.3,7.6$, and $10.7 \mathrm{~cm}$ downstream of the injector, respectively. All the tests were conducted in air.

Figure 16a shows an elongated plume, extending approximately $35 \mathrm{~mm}$ along $z$ and resembling the morphology of the injectors' trailing edge. In reality, the inspection of the numerical results presented in Sec. III reveals that the shape of the plume at this early stage of development is due to the initiated entrainment of fuel in the imposed vortical structures. Two distinct bulges having symmetry with respect to $z=16 \mathrm{~mm}$ begin to form, indicating areas of radical agglomeration. A filament of hydroxyl radicals connecting the plume to the wall is recognizable from $z=0$ to $4 \mathrm{~mm}$, approximately. Figure $16 \mathrm{c}$ shows a marked evolution of the morphology of the

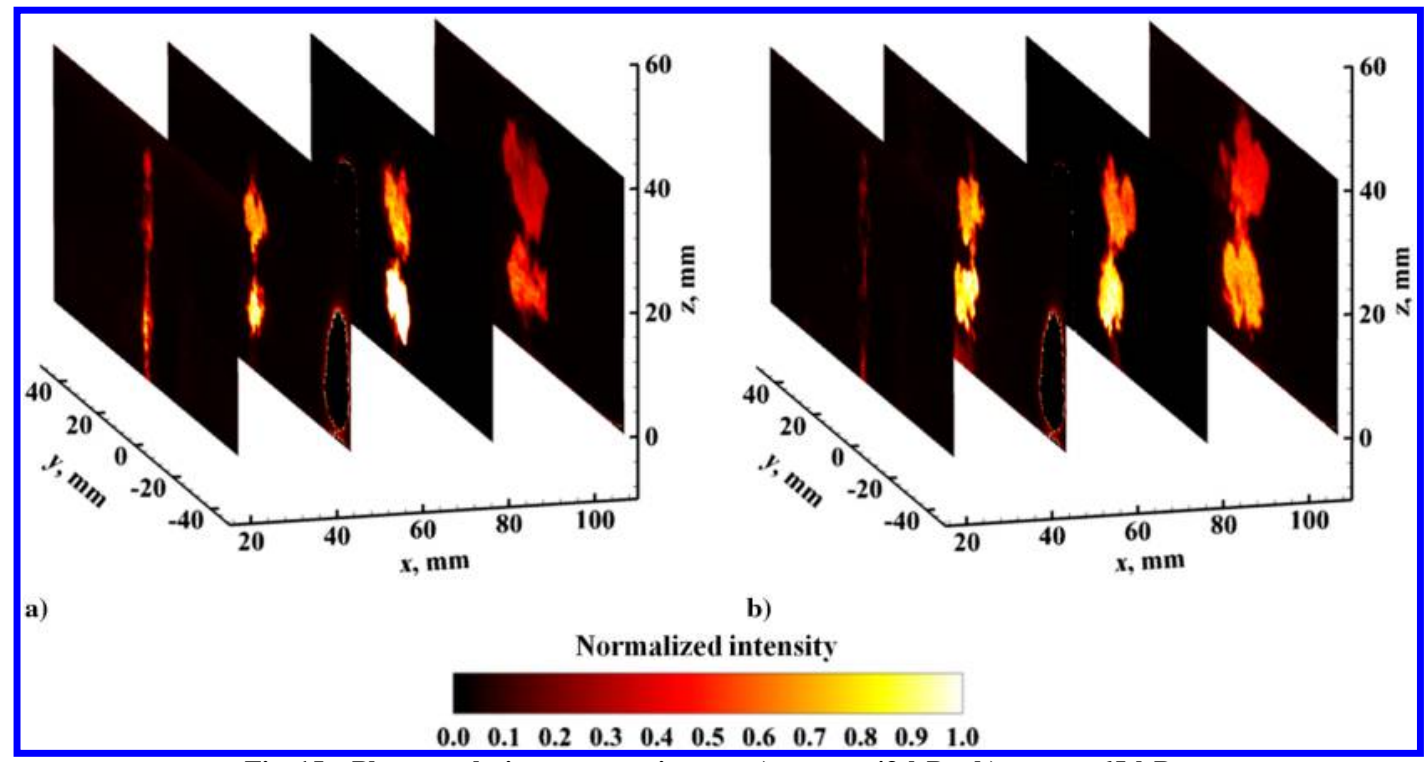

Fig. 15 Plume evolution, symmetric case: a) $p_{0, j 1}=43 \mathrm{kPa}$; b) $p_{0, j 2}=65 \mathrm{kPa}$. 

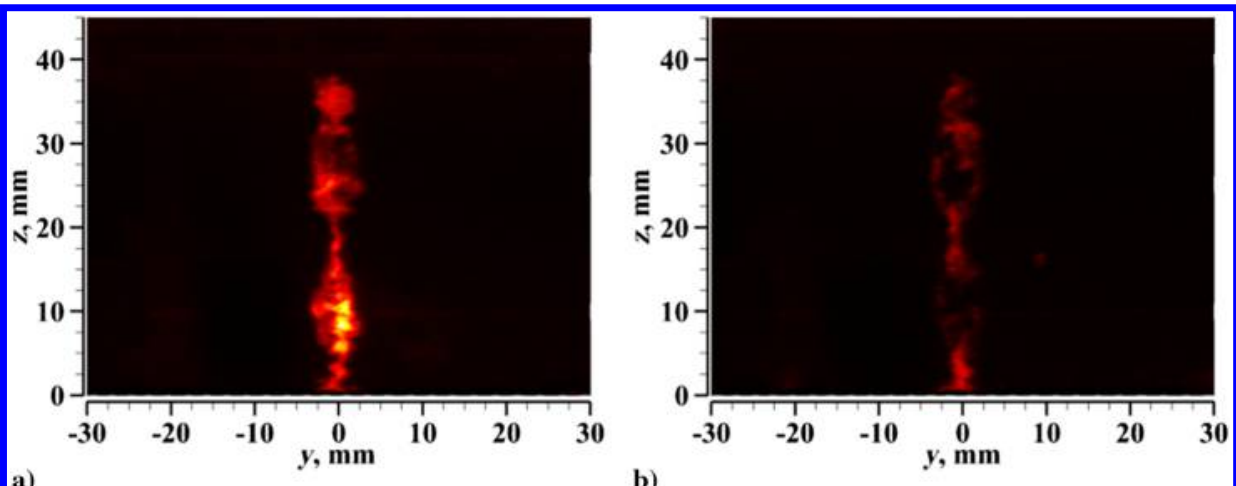

b)
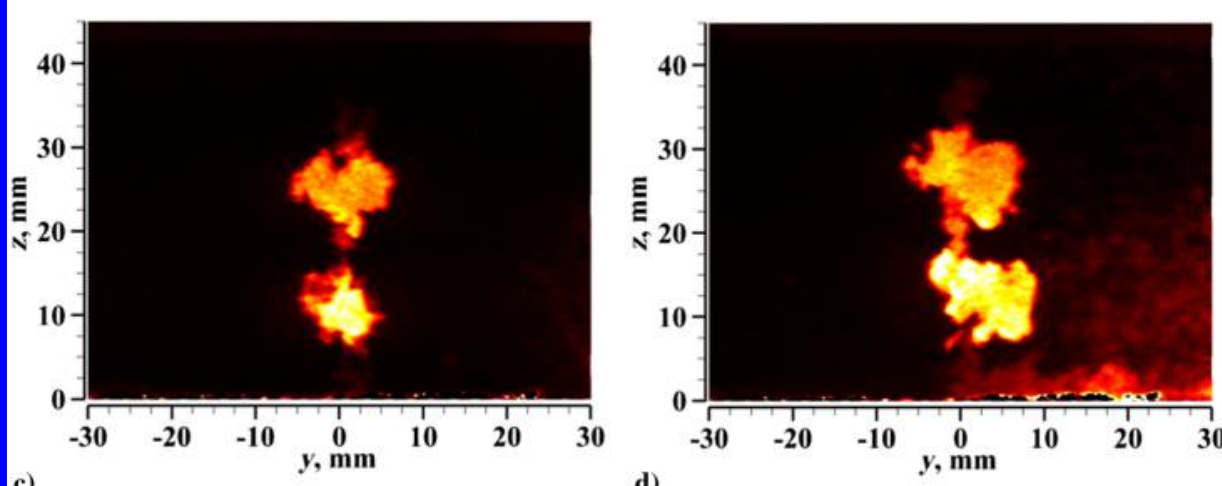

d)


h)

Normalized intensity

$\begin{array}{llllllllllllll}0.0 & 0.1 & 0.2 & 0.3 & 0.4 & 0.5 & 0.6 & 0.7 & 0.8 & 0.9 & 1.0\end{array}$

Fig. 16 OH PLIF instantaneous cross planes, symmetric case: left: $p_{0, j 1}=43 \mathrm{kPa}$; right: $p_{0, j 2}=65 \mathrm{kPa}$. a) and b) $\left.1.8 \mathrm{~cm}, \mathrm{c}\right)$ and d) $4.3 \mathrm{~cm}$, e) and f) $7.6 \mathrm{~cm}, \mathrm{~g}$ ) and h) $10.7 \mathrm{~cm}$.

plume: two distinct lobed-like areas can be seen to evolve from the smaller bulges, while still maintaining their level of symmetry about the $z=16 \mathrm{~mm}$ and $y=0 \mathrm{~mm}$ planes. At this section, the size of both lobes was such that each one could have been enclosed in a 10-mm-diam circle. Figure 16e shows a slight growth of the lobes, increasing the diameter of the circle required to enclose them to approximately $12 \mathrm{~mm}$. The lower lobe is characterized by a higher amount of $\mathrm{OH}$ radicals, qualitatively shown by the increased brightness in the area when compared with the upper lobe. The last section depicted in Fig. 16g, shows a broadening of the plume, 
which provides further evidence supporting the conclusion expressed in Sec. V.B. The lobes appear squeezed along the $z$ axis and spread along the $y$ direction reaching a width of approximately $20 \mathrm{~mm}$.

The set of image data in Figs. 16b, 16d, 16f, and 16h describe the plume evolution for the high-stagnation pressure case. Figure $16 \mathrm{~b}$ shows a thin plume similar to that seen in Fig. 16a, but characterized by lower amounts of $\mathrm{OH}$ radicals; nevertheless, the reaction zone is attached to the wall by a thin line of radicals similar to the result seen in Fig. 16a for the lower injection pressure case. Faster combustion at $4.3 \mathrm{~cm}$ downstream of the injection point (Fig. 16d) could be inferred from the agglomeration of radicals forming the two symmetric lobes already analyzed in Fig. 16c. In this section, a thin strip of $\mathrm{OH}$ radicals (approximately $2 \mathrm{~mm}$ in length) connected the lobes. This flow feature was also captured in each of the two other spanwise flowfields probed downstream (i.e., Figs. $16 \mathrm{f}$ and $16 \mathrm{~h}$ ), and this line did not present remarkable changes in length up to the last measurement plane. By comparing Figs. $16 \mathrm{f}$ and $16 \mathrm{~h}$, it is clear that the evolution of the plume is similar to that noted for the lower injection pressure case; that is, only a small variation in size can be observed, whereas the general shape of the lobes is maintained from station to station. Because of the increased fuel flow rate associated with the higher injection pressures, the two lobes can be seen to be slightly larger in Figs. 16d and 16h when compared with their lower pressure counterparts. Their general characteristic shapes, however, seem to be almost unaffected by the difference in injection conditions, despite the different amount of $\mathrm{OH}$ radicals in the plumes. The persistence of the lobes and the repetition of the same flow features in each section indicate that the coherent imposed structures were having an important effect on the dynamics driving the evolution of the plume. The thin filament of radicals connecting the plume to the wall is an interesting result. The presence of hydroxyl radicals in that region suggests that the recirculation expected at the base of the injector acted as a flameholding mechanism. However, further investigations should be addressed in clarifying this aspect because $\mathrm{OH}$ radicals are a long-lived species and it is possible that this area of concentration observed in the results is a consequence of the radicals being transported from elsewhere in the plume, rather than being a marker of combustion occurring directly on the wall.

\section{OH PLIF: Asymmetric Configuration}

The experimental outcomes for the asymmetric pylon are presented in Figs. 17 and 18. As can be seen, only three sections have been inspected for this configuration because the main scope of the investigation was to verify the predictions of the code concerning the plume morphology on a qualitative basis. Figure 17 indicates that the plume was highly distorted and kept a steady growth as it was flowing downstream.

Figures $18 \mathrm{a}$ and $\underline{18 \mathrm{~b}}(4.3 \mathrm{~cm}$ cross section) show a characteristic geometrical shape of the plume: a wide lobe and two smaller areas where $\mathrm{OH}$ agglomerated can be recognized in the middle and at the extremities, respectively. Apparently, the increased fuel flow rate in the high injection pressure case only slightly influenced both the height and the width of the plume. Detectable, and apparently regular, regions characterized by a lack of $\mathrm{OH}$ radicals are present in the center of the plume. This feature is here attributed to the local equivalence ratio which can be well above the ignition limits of the mixture. Based on the simulations presented in Sec. III, the vortex cores in the center of the plume were, in fact, expected to carry a larger amount of fuel compared with those on the top and the bottom as they migrate, spanning the width of the plume and entraining mainly hydrogen. A large amount of one reactant in the cores could lead to a nonignition condition in those areas, leaving the cores $\mathrm{OH}$ radicals free. This behavior is not present in the case of the symmetric configuration, where the vortex cores are expected to remain at the edges of the plume, entraining both air and fuel and providing ignition all across the plume closer to the jet exit plane.

Figures $18 \mathrm{c}$ and $18 \mathrm{~d}$ show the $7.6 \mathrm{~cm}$ station: here, the vortices were entraining fuel and air in the right proportions to ensure an homogeneous combustion and enhanced the plume spreading in their area of influence. The images taken at the last station (Fig. 18e and 18f) show a much larger combustion area in the central lobe. Although the main difference between the two injection pressures is the size of the lobe, which is slightly wider for the high injection pressure case, the position of the two peripheral structures, which can be inferred from the plume shape, remained nearly unchanged. As a consequence, the plume reaches a better spreading along both the $y$ and the $z$ axes if compared with the results obtained with the symmetric configuration. Also, in this asymmetric plume, $\mathrm{OH}$ radicals very close to the wall are recognizable in each image and indicate that a similar recirculation zone to that noticed in the previous case (see Sec. V.C) was present downstream of the injection slit, providing favorable conditions for ignition and flameholding.

\section{Plume Evolution Comparisons}

The experimental results showed noticeable analogies with the simulations presented in Sec. III. It is important to remind the reader that, although the instantaneous images collected may not be representative of an average behavior of the flow, the repeatability of

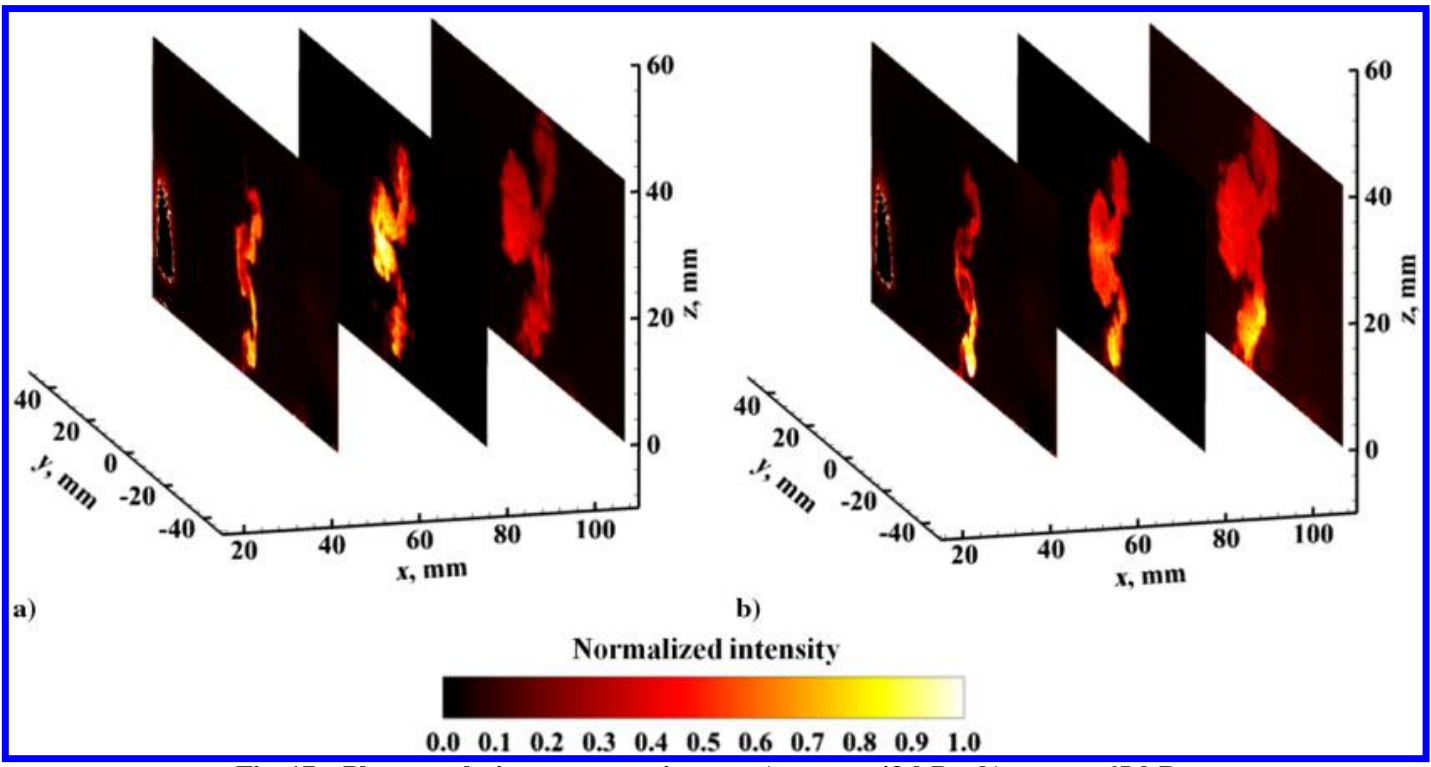

Fig. 17 Plume evolution, asymmetric case: a) $p_{0, j 1}=43 \mathrm{kPa}$; b) $p_{0, j 2}=65 \mathrm{kPa}$. 

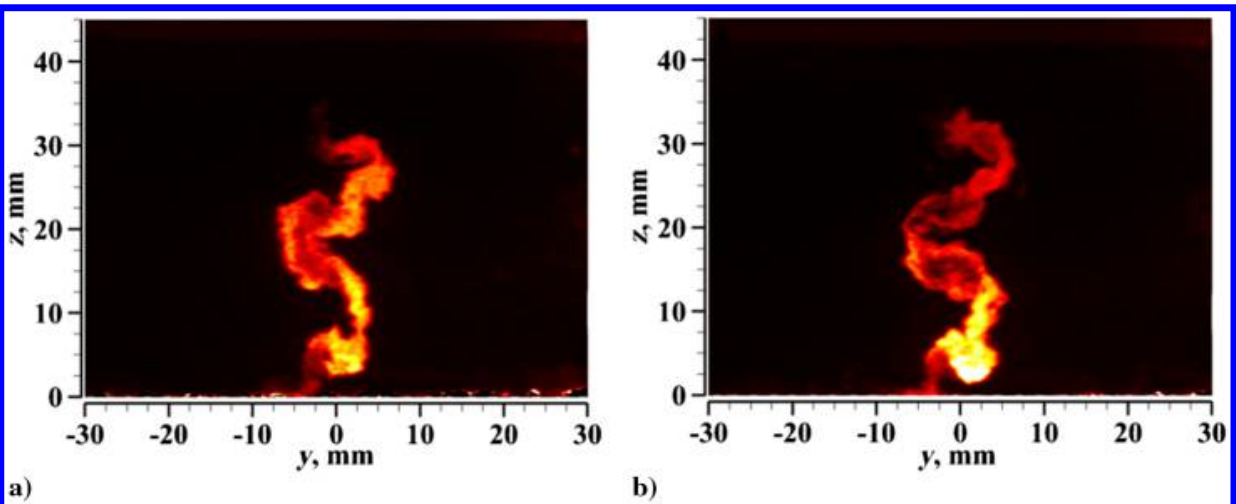

a)

b)
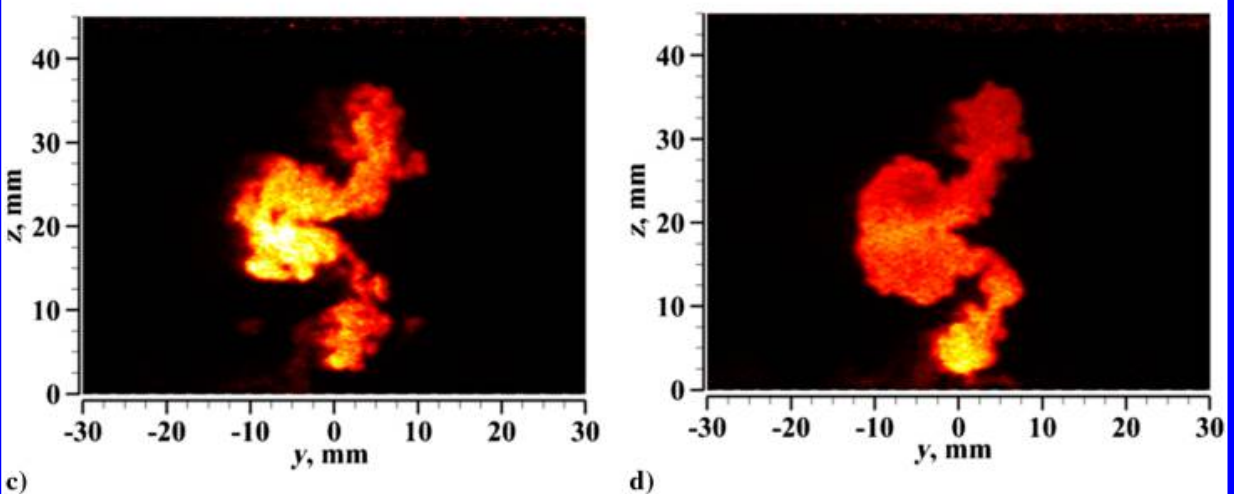

c)

d)
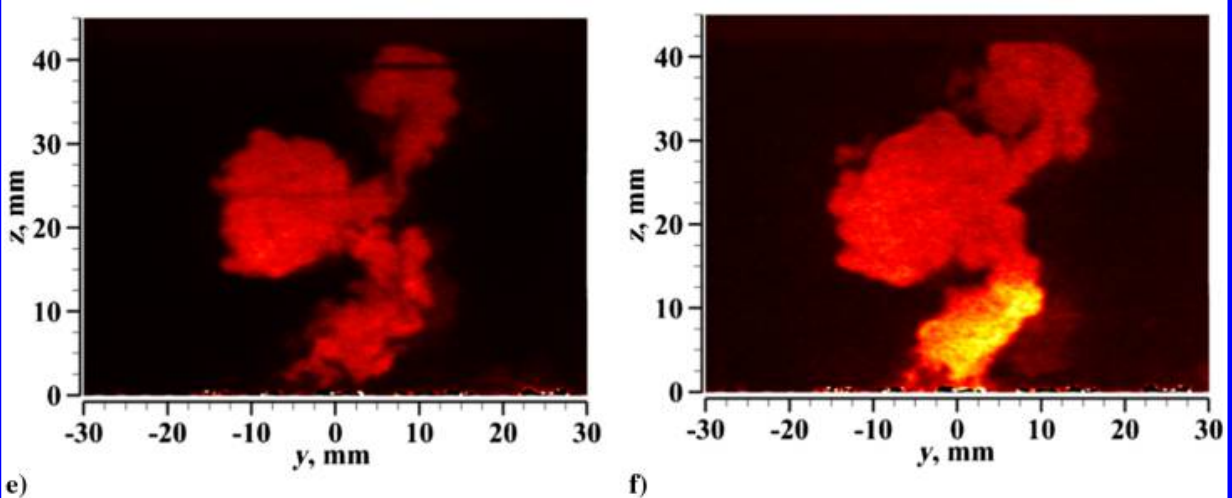

Normalized intensity

$\begin{array}{lllllllllll}0.0 & 0.1 & 0.2 & 0.3 & 0.4 & 0.5 & 0.6 & 0.7 & 0.8 & 0.9 & 1.0\end{array}$

Fig. 18 OH PLIF instantaneous cross planes, asymmetric case: left: $p_{0, j 1}=43 \mathrm{kPa}$; right: $p_{0, j 2}=65 \mathrm{kPa}$. a) and b) $\left.4.3 \mathrm{~cm}, \mathrm{c}\right)$ and d) $7.6 \mathrm{~cm}$, e) and f) $10.7 \mathrm{~cm}$.

the plume's geometrical features at each station and along the probed flowfield by means of uncorrelated measurements are evident proofs that coherent structures acted to modify the flowfield as predicted.

In the simulations conducted for the symmetric case, the vortical structures evolving from the ramps interacted, generating two distinct quadruplets of vortices as they moved downstream. The agglomeration of streamwise vortices in the two areas was the cause of the lobed plume similar to that experimentally captured in the OH PLIF data images, as can be seen in Fig. 19, where a direct comparison of the experimental and numerical results obtained at all the stations is presented. Although the absolute lateral spreading of the plume was not fully captured, Fig. 19 reveals the existence of the double-lobed plume already predicted by the simulation. In addition, some of the details recognizable in the experiments are present in the numerical simulations: For example, the numerical simulations show a thin vertical segment of tracers connecting the lobes, clearly resembling the strip of $\mathrm{OH}$ radicals experimentally visualized between the bulges. Also, the early development of the experi- mentally surveyed plume is attributable to the initial entrainment of fluid in the vortical structures, as suggested by Fig. $19 \mathrm{~b}$.

The experimental plume and the calculated dispersion of virtual tracers for the asymmetric pylon injector is presented in Fig. 20. Also in this case, the code predicted very closely the overall shape of the issued plume. The markers show a wider dispersion along both the $y$ and $z$ axes when compared with the symmetric case, confirming the outcome of the experimental findings. In the numerical simulations, two mergers were expected to form in the center of the plume due to the coalescence of vortical structures of the same sign: In this way, the circulation increased in a relatively narrow zone of the flow, localizing most of the entrainment of tracers in the same region. The remaining external vortices, on the other hand, kept their straight trajectory nearly undisturbed (see Fig. 5). When comparing the plume geometry obtained in the experiments and the numerical results, it is clear that the combination of the two structures formed in the center and those convecting downstream at the upper and lower edges of the plume acted to shape it as both predicted and measured. 

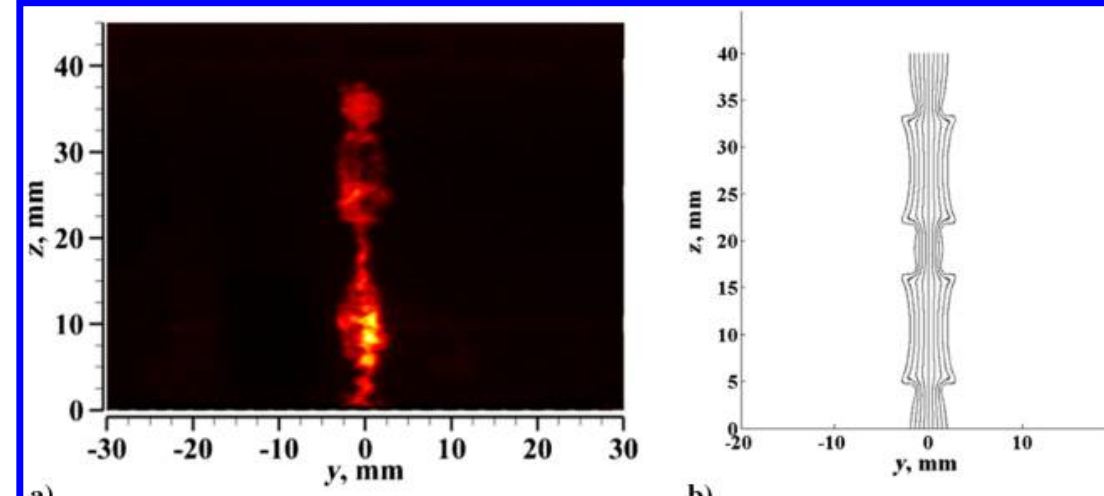

b)
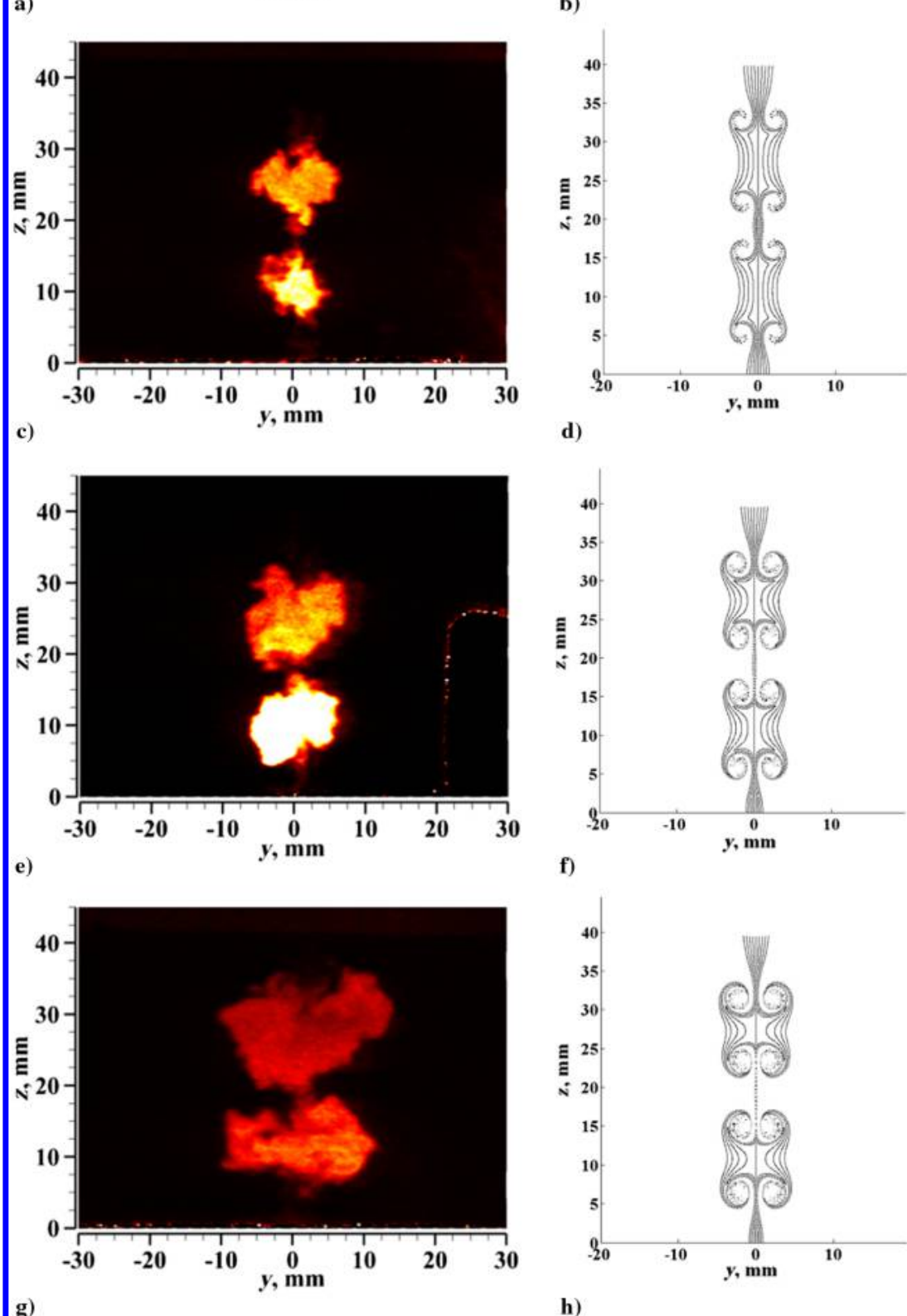

d)



f)

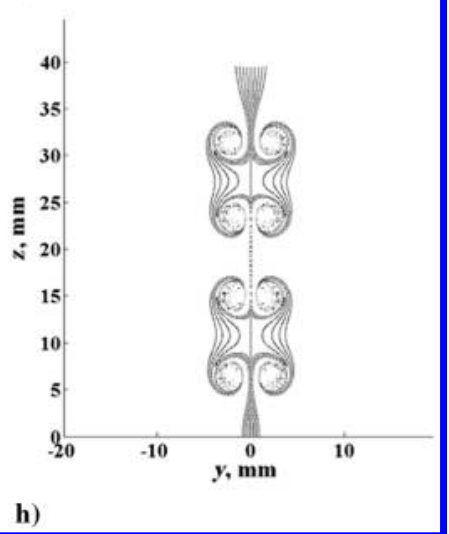

Fig. 19 Comparison between experimental and numerical plumes, symmetric case. Left: experiment; right: simulation. a) and b) $1.8 \mathrm{~cm}$, c) and d) $4.3 \mathrm{~cm}$, e) and f) $7.6 \mathrm{~cm}, \mathrm{~g}$ ) and h) $10.7 \mathrm{~cm}$.

As already mentioned in Sec. V.D, some of the areas in the center of the plume captured at the streamwise station located $4.3 \mathrm{~cm}$ downstream of the injection plane are characterized by a lower amount of radicals compared with the peripheral regions (see Figs. $18 \mathrm{~b}$ and 20a). The explanation of this additional plume feature can also be attributed to the vortex interaction mode considered.
The simulations presented in Sec. III showed that two of the cores of the merging vortices in the center of the plume would have spanned the entire width of the pylon crossing the injection center plane in the early stage of their interaction (see Fig. 5), thus carrying an amount of fuel that likely led to a nonignition condition in those areas, leaving the cores nearly $\mathrm{OH}$ radicals free. 


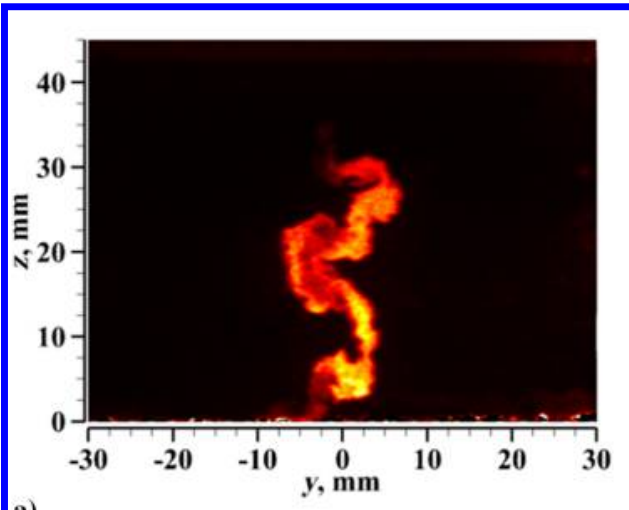

a)

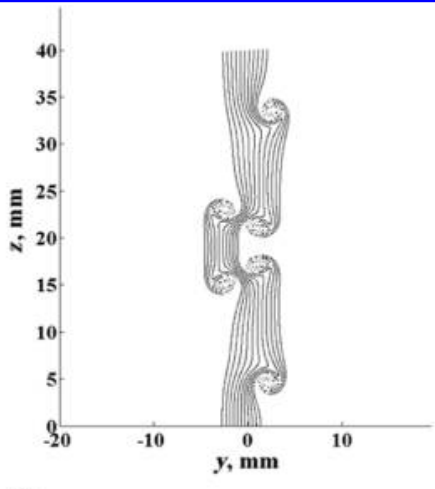

b)
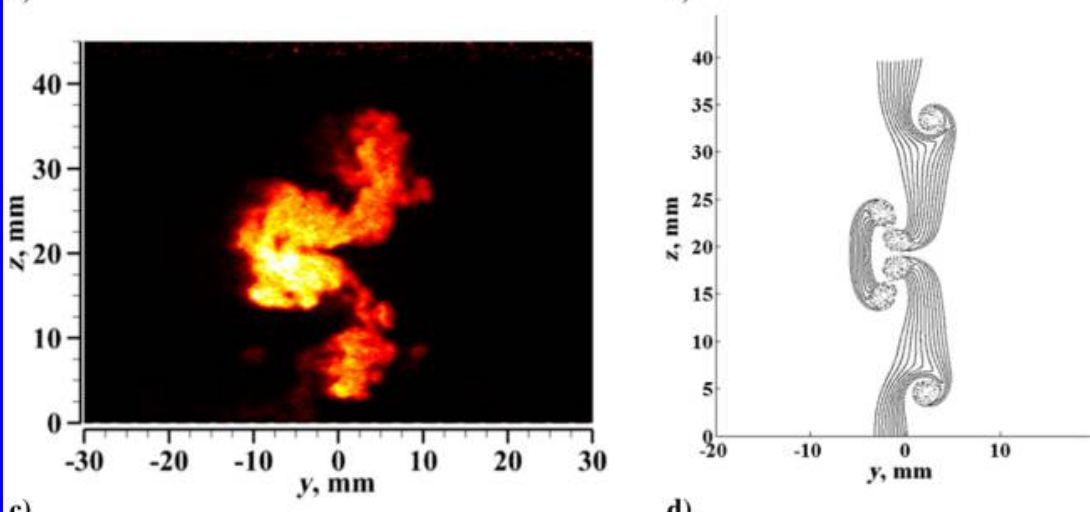

d)
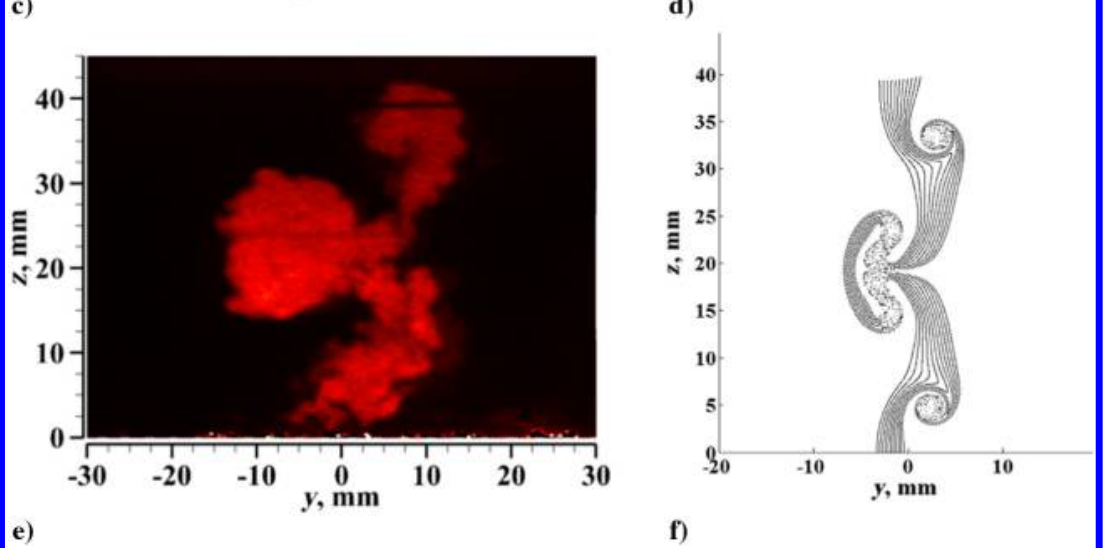

Fig. 20 Comparison between experimental and numerical plumes, asymmetric case. Left: experiment; right: simulation. a) and b) $4.3 \mathrm{~cm}$, c) and d) $7.6 \mathrm{~cm}$, e) and f) $10.7 \mathrm{~cm}$.

\section{Conclusions}

The supersonic combustion results of pylon-injected hydrogen in high-enthalpy flow with imposed vortex dynamics have been presented in this manuscript. Based on the simulations conducted with a reduced-order model developed by the University of Texas at Arlington research group, two different imposed plume evolutions, resulting from selected vortex dynamics modes, have been studied. The first vortex interaction mode was designed to generate two distinct quadruplets of streamwise vortical structures, evolving to produce a double-lobed plume in which limited lateral spreading was expected. In the second scenario, six vortices (three CVPs) were arranged to generate a single-lobed plume intended to augment the spreading along the lateral direction due to the merging process of two pairs of corotating vortices. In both cases, the strength of the vortical structures has been selected to produce the expected morphology within the limited streamwise extent (approximately $15 \mathrm{~cm}$ ) of the optically observable flowfield offered by the test arrangement of the expansion tube facility at the High-Temperature Gas Dynamics Laboratory of Stanford University, where the experiments were later conducted.

For the experimental campaign, two injectors were designed based on the outcomes of the simulations and rapid prototyped with a high- performance resin. The final geometry of the injectors resulted in two pylons with the same base configuration, but equipped with two different sets of vortex generators. The chosen vortex generators were ramps that have been integrated into the design of the pylons and specifically sized and placed to reproduce the desired vortex interaction scenarios.

Time-resolved schlieren, time-averaged $\mathrm{OH}^{*}$ chemiluminescence, and $\mathrm{OH}$ PLIF measurements have been used to document the combustion characteristics and evolution of the reacting plume associated with each injector. Specifically, $\mathrm{OH}^{*}$ chemiluminescence documented the ignition characteristics of the injectors; the results highlighted that autoignition of hydrogen took place in very close proximity of the injection point and the reacting region was confined within the optically accessible portion of the test section. No physical flameholding device was used. Subsequently, the OH PLIF survey allowed a detailed inspection of the reacting plume morphology across several selected streamwise stations. It revealed the presence of an evolving double-lobed plume structure for the first injector and an evolving triple-lobed plume for the second. These conditions were found for two different injection total pressures. The similarity of the plume morphology revealed in all the uncorrelated $\mathrm{OH}$ PLIF instantaneous images acquired at each probed section is a 
clear indication of the persistence of the streamwise vortical structures shed from the ramps and their ability to shape the resulting flowfield

The OH PLIF image data compared very well with the plume morphology predicted by the numerical simulations based on the reduced-order model. Specifically, the experimental results associated with the first pylon show the same double-lobed plume morphology shown in the simulations, and the results from the second injector have been found to be consistent with the predicted triple-lobed plume. Therefore, for the heat release levels obtained under these investigations, the conclusion that can be drawn is that plume morphology was shaped in accordance with the evolution of the dominant streamwise vortical structures in the flow.

These results are encouraging because of the intrinsic potential related to the design of new injection strategies suitable for scramjet combustors and based on the ability to predict, with a highly reduced computational cost, the main features of these complex flows. In addition, this vortex dynamics perspective will allow exploration of integrated configurations where inlet vortices could be used in conjunction with those introduced by the injection system for mixing and combustion enhancement.

\section{References}

[1] Papamoschou, D., and Roshko, A., "Compressible Turbulent Shear Layer: An Experimental Study," Journal of Fluid Mechanics, Vol. 197, Dec. 1988 , pp. $453-477$ doi:10.1017/S0022112088003325

[2] Chigier, N. A., and Chervinsky, A., "Experimental Investigation of Swirling Vortex Motion in Jets," Journal of Applied Mechanics, Vol. 34, No. 2, 1969, pp. 443-451. doi: $10.1115 / 1.3607703$

[3] Swithenbank, J., and Chigier, N. A., "Vortex Mixing for Supersonic Combustion," XII International Symposium on Combustion, Combustion Inst., Pittsburgh, PA, 1969, pp. 1152-1162.

[4] Waitz, I. A., Qiu, Y. J., Manning, T. A., Fung, A. K. S., Elliot, J. K., Kerwin, J. M., Krasnodebski, J. K., O'Sullivan, M. N., Tew, D. E., Greitzer, E. M., Marble, F. E., Tan, C. S., and Tillman, T. G., "Enhanced Mixing with Streamwise Vorticity," Progress in Aerospace Sciences, Vol. 33, Nos. 5-6, 1997, pp. 323-351. doi:10.1016/S0376-0421(96)00008-5

[5] Yang, J., Kubota, T., and Zukoski, E. E., "Applications of ShockInduced Mixing to Supersonic Combustion," AIAA Journal, Vol. 31, No. 5,1993 , pp. 854-862. doi: $10.2514 / 3.11696$

[6] Naughton, J. W., Cattafesta, L. N., III, and Settles, G. S., "Experimental Study of Compressible Turbulent Mixing Enhancement in Swirling Jets," Journal of Fluid Mechanics, Vol. 330, Jan. 1997, pp. 271-305. doi:10.1017/S0022112096003679

- [7] Marble, F. E., "Growth of a Diffusion Flame in the Field of a Vortex," Recent Advances in the Aerospace Sciences, Plenum, New York, 1985, pp. 395-413.

[8] Waitz, A., Marble, F. E., and Zukoski, E. E., "Vorticity Generation by Contoured Wall Injectors," AIAA Paper 1992-3550, 1992.

-[9] Keffer, J. F., and Baines, W. D., "Round Turbulent Jet in a Cross-Wind," Journal of Fluid Mechanics, Vol. 15, No. 4, 1963, pp. 481-496. doi:10.1017/S0022112063000409

[10] Campbell, J. F., and Schetz, J. A., "Analysis of the Injection of a Heated Turbulent Jet into a Cross Flow," NASA TR-413, 1973.

[11] Billig, F., and Schetz, J. A., "Analysis of Penetration and Mixing of Gas Jets in Supersonic Cross Flow,” AIAA Paper 1992-5061, 1992.
[12] Marble, F. E., Zukoski, E. E., Jacobs, J. W., Hendricks, G. J., and Waitz, I. A., "Shock Enhancement and Control of Hypersonic Mixing and Combustion," AIAA Paper 1990-1981, 1990.

[13] Maddalena, L., "Investigations of Injectors for Scramjet Engines," Ph.D. Dissertation, Virginia Polytechnic Inst. and State Univ., Blacksburg, VA, 2007.

[14] Vergine, F., and Maddalena, L., "Evolution of Large-Scale Structures Generated by a Strut Injector in a Mach 2.5 Flow," AIAA Paper 20120332,2012

[15] Vergine, F., Crisanti, M., and Maddalena, L., "Investigation of the Merging Process and Dynamics of Streamwise Vortices Generated by a Flow-Mixing Device in a Mach 2.5 Flow," AIAA Paper 2013-0699, 2013.

[16] Tam, C. J., Hsu, K. Y., Gruber, M. R., and Raffoul, C. N., "Fuel/Air Mixing Characteristics of Strut Injections for Scramjet Combustor Applications," AIAA Paper 2008-6925, 2008.

[17] Hsu, K. Y., Carter, C. D., Gruber, M. R., and Tam, C. J., "Mixing Study of Strut Injectors in Supersonic Flows," AIAA Paper 2009-5226, 2009.

[18] Doster, J. C., King, P. I., Gruber, M. R., Carter, C. D., Ryan, M. D., and Hsu, K., "In-Stream Hypermixer Fueling Pylons in Supersonic Flow," Journal of Propulsion and Power, Vol. 25, No. 4, 2009, pp. 885-901. doi:10.2514/1.40179

[19] Sunami, T., Magre, P., Bresson, A., Grisch, F., Orain, M., and Kodera, M., "Experimental Study of Strut Injectors in a Supersonic Combustor Using OH-PLIF," AIAA Paper 2005-3304, 2005.

[20] Maddalena, L., Vergine, F., and Crisanti, M., "Vortex Dynamics Studies in Supersonic Flow: Merging of Co-Rotating Streamwise Vortices," Physics of Fluids, Vol. 26, No. 4, 2014, Paper 046101. doi: $10.1063 / 1.4871022$

[21] Ferri, A., Elements of Aerodynamics of Supersonic Flows, Macmillan, New York, 1949, pp. 350-359.

[22] Smart, M. K., Kalkhoran, I. M., and Popovic, S., "Some Aspects of Streamwise Vortex Behavior During Oblique Shock Wave/Vortex Interaction," Shock Waves, Vol. 8, No. 4, 1998, pp. 243-255. doi:10.1007/s001930050117

[23] Klaas, M., Schroder, W., and Althaus, W., "Experimental Investigation of Slender Streamwise Vortices and Oblique Shock-Vortex Interaction," AIAA Paper 2005-4652, 2005.

[24] Jeong, J., and Hussain, F., "On the Identification of a Vortex," Journal of Fluid Mechanics, Vol. 285, Feb. 1995, pp. 69-94. doi:10.1017/S0022112095000462

[25] Heltsley, W. N., Snyder, J. A., Houle, A. J., Davidson, D., Mungal, M. G., and Hanson, R. K., "Design and Characterization of the Stanford 6 Inch Expansion Tube," AIAA Paper 2006-4443, 2006.

[26] Ben-Yakar, A., and Hanson, R., "Cavity Flame-Holders for Ignition and Flame Stabilization in Scramjets: An Overview," Journal of Propulsion and Power, Vol. 17, No. 4, 2001, pp. 869-877. doi: $10.2514 / 2.5818$

[27] Najm, H., Paul, P., Mueller, C., and Wyckoff, P., "On the Adequacy of Certain Experimental Observables as Measurements of Flame Burning Rate," Journal of Combustion and Flame, Vol. 113, No. 3, 1998, pp. 312-332. doi:10.1016/S0010-2180(97)00209-5

[28] Haber, L. C., Vandsburger, U., Saunders, W. R., and Khanna, V. K., "Experimental Examination of the Relationship Between Chemiluminescent Light Emissions and Heat-Release Rate Under Non-Adiabatic Conditions," Defense Technical Information Center Compilation Part Notice ADP011163, Fort Belvoir, VA, 2001.

L. Maurice Associate Editor 
This article has been cited by:

1. Yasin M. Abul-Huda, Mirko GambaEnhanced Combustion in Supersonic Flows Using a Pulsed Detonation . [Citation] [PDF] [PDF Plus] 